\title{
Temporal Dynamics of Sensorimotor Networks in Effort-Based Cost-Benefit Valuation: Early Emergence and Late Net Value Integration
}

\author{
๑Alison Harris ${ }^{1}$ and ${ }^{\circledR S}$ Seung-Lark Lim ${ }^{2}$ \\ ${ }^{1}$ Claremont McKenna College, Claremont, California 91711, and 2University of Missouri-Kansas City, Kansas City, Missouri 64110
}

\begin{abstract}
Although physical effort can impose significant costs on decision-making, when and how effort cost information is incorporated into choice remains contested, reflecting a larger debate over the role of sensorimotor networks in specifying behavior. Serial information processing models, in which motor circuits simply implement the output of cognitive systems, hypothesize that effort cost factors into decisions relatively late, via integration with stimulus values into net (combined) value signals in dorsomedial frontal cortex ( $\mathrm{dmFC}$ ). In contrast, ethology-inspired approaches suggest a more active role for the dorsal sensorimotor stream, with effort cost signals emerging rapidly after stimulus onset. Here we investigated the time course of effort cost integration using event-related potentials in hungry human subjects while they made decisions about expending physical effort for appetitive foods. Consistent with the ethological perspective, we found that effort cost was represented from as early as $100-250 \mathrm{~ms}$ after stimulus onset, localized to dorsal sensorimotor regions including middle cingulate, somatosensory, and motor/premotor cortices. However, examining the same data time-locked to motor output revealed net value signals combining stimulus value and effort cost approximately $-400 \mathrm{~ms}$ before response, originating from sensorimotor areas including $\mathrm{dmFC}$, precuneus, and posterior parietal cortex. Granger causal connectivity analysis of the motor effector signal in the time leading to response showed interactions between these sensorimotor regions and ventrolateral prefrontal cortex, a structure associated with adjusting behavior-response mappings. These results suggest that rapid activation of sensorimotor regions interacts with cognitive valuation systems, producing a net value signal reflecting both physical effort and reward contingencies.
\end{abstract}

Key words: decision-making; effort; ERP; sensorimotor

Significance Statement

Although physical effort imposes a cost on choice, when and how effort cost influences neural correlates of decision-making remains contested. This dispute reflects a larger disagreement between cognitive neuroscience and ethology over the role of sensorimotor systems in behavior: are sensorimotor circuits merely implementing the late-stage output of cognitive systems, or engaged rapidly and interactively from early in decision-making? We find that, although early representation of effort cost is associated with sensorimotor regions, these signals are also integrated with cognitive stimulus value representations in the time leading up to motor response. These data suggest that sensorimotor networks interact dynamically with cognitive systems to guide decision-making, providing a first step toward reconciling differing perspectives on sensorimotor roles in valuation and choice.

\section{Introduction}

Physical effort, conceptualized as the cost associated with action to obtain an outcome (Rangel and Hare, 2010), often affects our

\footnotetext{
Received Nov. 6, 2015; revised May 20, 2016; accepted May 25, 2016.

Author contributions: A.H. and S.-L.L. designed research; A.H. performed research; A.H. analyzed data; A.H. and S.-L.L. wrote the paper.

We thank Antonio Rangel for supporting this research and Catherine L. Reed and Joo-Hyun Song for their helpful comments on a previous version of this manuscript.

The authors declare no competing financial interests.

Correspondence should be addressed to Dr. Alison Harris, Department of Psychology, Claremont McKenna Col-

lege, 850 Columbia Avenue, Claremont, CA 91711. E-mail: aharris@cmc.edu.
}

choices. However, when and how physical effort influences decision-making at the neural level is debated, reflecting tensions between cognitive neuroscience and ethological approaches over sensorimotor contributions to valuation and choice.

Inspired by information processing models (Sternberg, 1969), cognitive researchers have proposed a serial progression from perceptual and cognitive stages to motor output, into which effort cost enters relatively late. In this framework, cognitive valu- 
ation areas, such as ventromedial prefrontal cortex (vmPFC), first compute and compare the values of different options (Kable and Glimcher, 2009; Clithero and Rangel, 2014), with the choice outcome then passed to action selection regions for transformation into motor output (Padoa-Schioppa, 2011). Candidates for this good-to-action transformation include anterior cingulate cortex (ACC) and dorsomedial frontal cortex (dmFC), which encode information not only about the intrinsic value of rewards but also the effort required to obtain them (Croxson et al., 2009; Prévost et al., 2010; Kennerley et al., 2011; Rushworth et al., 2011; Burke et al., 2013). With activity correlated with net value (stimulus value minus effort cost) and rich connectivity to motor control systems (Paus, 2001), these areas may contribute to translating cognitive representations of value into specific actions (Wunderlich et al., 2009).

In contrast, ethology-based models, such as the affordance competition hypothesis (Cisek and Kalaska, 2010), suggest that sensorimotor networks may represent effort cost in a parallel and interactive manner during decision-making (Song and $\mathrm{Na}$ kayama, 2009; Cisek, 2012). In this view, information relevant to possible actions is represented in the dorsal sensorimotor stream from early in the decision process, with the choice of action then refined via inputs from areas encoding goal-relevant variables. Consistent with this idea, neurons in posterior parietal cortex (Thomas and Paré, 2007) and premotor cortex (Cisek and Kalaska, 2005) can represent potential saccade targets or movements, respectively, as early as $50-100 \mathrm{~ms}$ after cue onset, well before the implementation of a motor response.

Therefore, these two models differ in their predictions regarding when sensorimotor networks represent physical effort cost and how effort cost is transformed into motor output. Although the information processing model is agnostic to the timing of effort cost computations, its late integration of net value suggests that sensorimotor involvement should be mostly confined to the action selection period. In contrast, the affordance competition hypothesis makes specific predictions about the initial time course of effort cost processing, but it is unclear whether this system would require the computation of an explicit net value signal.

Reconciling these models has proven difficult, in part, because of technical tradeoffs between experimental approaches, including the limited number of recording sites in electrophysiological methods and the poor temporal resolution of fMRI. Here we used event-related potentials (ERPs) combined with advanced statistical and source localization techniques while hungry human subjects decided about expending physical effort to obtain appetitive foods. With millisecond temporal resolution and wholehead coverage, this approach allowed us to map how effort cost and stimulus value computations emerged and interacted during decision-making.

First, we examined the data time-locked to the onset of food and effort cues. The affordance competition hypothesis would predict rapid effort cost processing (e.g., 100-200 ms after stimulus onset), localized to sensorimotor regions. Based on previous data (Harris et al., 2011, 2013), we hypothesized that stimulus value would be represented later, $\sim 450-650 \mathrm{~ms}$ after stimulus onset, in sources including vmPFC. In contrast, integrated net value signals in regions such as ACC and dmFC are hypothesized to reflect the transformation of stimulus value into action (Padoa-Schioppa, 2011). Therefore, because of the large variability of action transformation timing across trials, it is unclear whether net value signals would be visible in the stimuluslocked data. Instead, we may observe net value signals only when
A
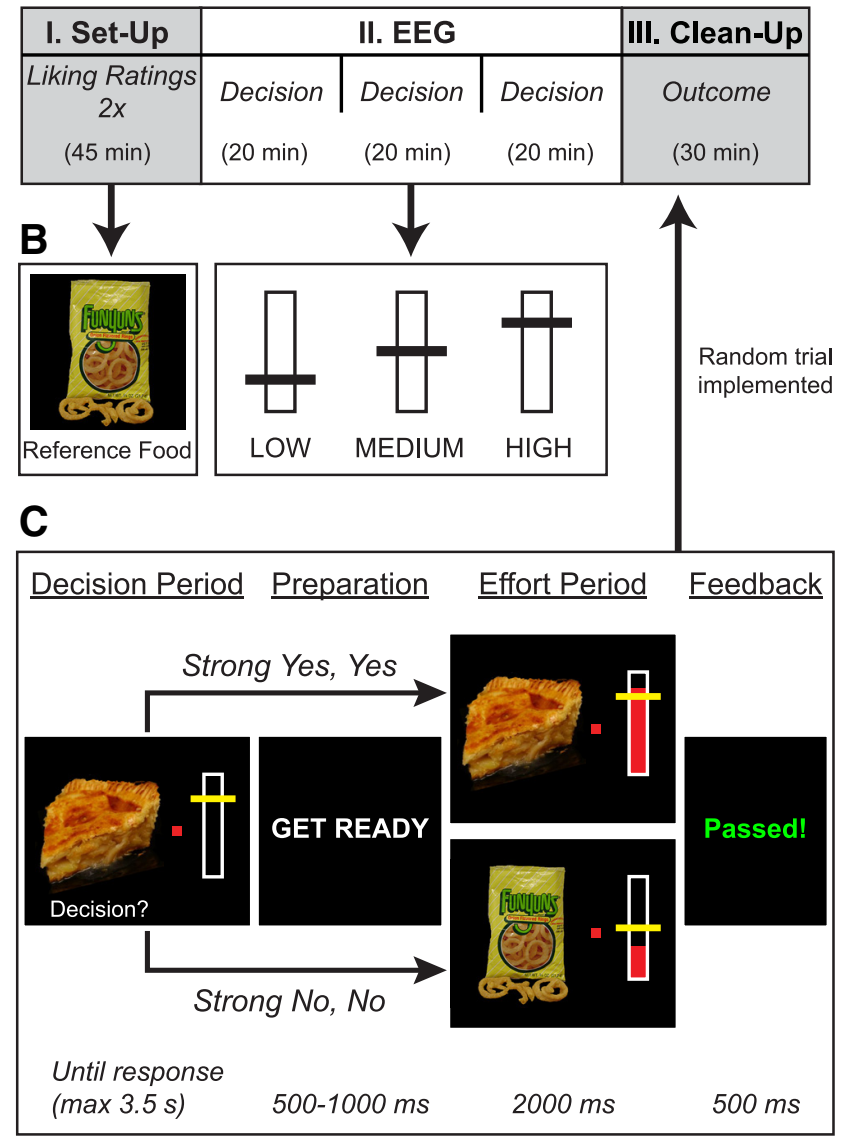

Figure 1. Experimental procedure. $A$, The experiment consisted of three parts: Part I, behavioral liking ratings, repeated in two blocks to obtain the average liking rating for each item; Part II, in which subjects decided whether to exert effort for the chance to obtain a food at the end of the experiment, during which their brain activity was recorded with EEG; and Part III, in which the outcome of a randomly selected trial was implemented depending on the subject's decision and successful exertion of effort. $\boldsymbol{B}$, Left, On every trial, subjects chose between working for the depicted food versus working for a reference food, randomly selected from the set of items assigned a neutral rating (2.5 of 4). Right, All other foods were paired with three different levels of effort (low, medium, high), corresponding to 25,50 , and $75 \%$ of maximum grip strength. The reference food was always paired with medium effort. $C$, Schematic of a trial from the decision task. On each trial, participants saw a snack food paired with one of three effort costs and responded with whether they wished to expend the specified effort for the chance to obtain the depicted food (strong no to strong yes) versus expending medium effort for the reference food. After their response and a short preparatory period, they then expended effort while receiving real-time feedback about their grip strength. At the end of the experiment, a single trial was randomly selected for the outcome phase in Part III.

ERPs are time-locked to motor output, reflecting the role of these computations in preparing the key-press response.

\section{Materials and Methods}

Subjects. Data collection took place at the California Institute of Technology (Caltech) in Pasadena, CA. Twenty-seven right-handed subjects (aged 18-40 years, 17 males) from the local community participated in the study. Two individuals were excluded for excess number of noisy electroencephalography (EEG) electrodes and/or failing to use the full range of the liking rating scale. All subjects provided written informed consent before participation, and all procedures were reviewed and approved by the Caltech Institutional Review Board.

Stimuli. Subjects viewed color pictures of 200 foods on a black background (Fig. 1; $432 \times 432$ pixels). The set of foods consisted of common appetitive snacks, including fruits, chips, and candy bars. During the decision task, each food was paired with a "thermometer bar" consisting 
of a white rectangle with a horizontal yellow bar indicating the level of effort (low, medium, high) associated with the trial. Relative to a central fixation point, the food was always presented on the left side of the screen, with the effort bar on the right. Size and eccentricity of the food and effort bar stimuli were adjusted to ensure ease of identification while maintaining central fixation. In the effort period, real-time feedback about grip strength was provided via a dynamically fluctuating red rectangle within the thermometer bar; as described below, subjects had to monitor and adjust their grip strength to maintain the red bar at a height at or above the yellow line indicating the desired effort level.

Procedure. In this experiment, subjects decided whether to expend various degrees of physical effort for different appetitive foods. Physical exertion was measured using a BIOPAC MP-150 system with a MRcompatible hand clench dynamometer transducer (TSD121B-MRI; BIOPAC Systems). Analog voltage measures of grip strength were transmitted to MATLAB (MathWorks) for real-time feedback using the PsychHID interface in the Psychophysics Toolbox (Brainard, 1997). Throughout the entire experiment, handgrip responses were made with the dominant hand, and button-press responses were recorded on a keyboard using the left hand.

Subjects were asked to fast for at least $3 \mathrm{~h}$ before the experiment. The experimental session consisted of three parts (Fig. 1) involving both behavioral ratings and EEG. In Part I, subjects indicated their preferences for a variety of snack foods. In Part II, subjects decided whether they wanted to expend a specified amount of effort for the chance to obtain each food at the end of the experiment while their brain activity was recorded with EEG. On each trial, this decision was immediately followed by an effort period in which the subject exerted the specified effort for the pictured food or a medium level of effort for a default "reference food" option. In Part III, the outcome of a randomly selected trial was implemented, with the subject receiving the specified food only if he had successfully exerted the required effort.

For Part I, subjects completed two blocks of trials in which they indicated their preferences for the full set of 200 foods, for a total of 400 ratings. Each food was displayed onscreen until the subject responded. Responses were measured using a four-point scale (1, strong no; 2 , no; 3 , yes; 4 , strong yes), entered via key press using the left hand. The order of the scale (left-to-right or right-to-left) was counterbalanced across subjects. By averaging the two sets of responses for each food, we obtained a set of liking ratings from 1 to 4 in 0.5 unit increments. From these ratings, we identified a subset of neutral items for each subject (liking rating of 2.5), consisting of items the subject neither liked nor disliked. Subjects were instructed that a single neutral item from their ratings would be randomly selected to serve as the reference food for the decision portion of the experiment (Fig. 1B, left).

In Part II, EEG data were measured while subjects performed a simple decision-making task in which they exerted effort by squeezing a hand dynamometer for the chance to obtain foods. Before the start of the task, we calibrated the hand dynamometer by instructing subjects to squeeze as hard as possible. Although the responses were measured in arbitrary units, the resulting measure of grip strength was compared with grip strength data collected from pilot subjects to ensure that subjects were in fact exerting the requested physical effort. If a subject's initial calibration number appeared low, the calibration was repeated until a reasonable estimate was obtained. Recalibration was performed at the beginning of each subsequent block of trials to account for reductions in grip strength over time attributable to muscle fatigue and hand soreness, for a total of three calibrations per subject. Because subjects tended toward overenthusiasm in attempting their maximum grip strength during the calibration, the "maximum grip strength" was computed as $90 \%$ of the measured calibration value, reducing the potential negative consequences of having an excessively high initial maximum value over many runs of trials. We then derived individual measures of low, medium, and high effort, corresponding to 25,50 , and $75 \%$ of the maximum grip strength (Fig. $1 B$, right).

Figure $1 C$ depicts the time course of a single trial during the decision task in Part II. On each trial, subjects saw one of the 200 foods paired with one of three effort levels (low, medium, high). Over the course of the experiment, each food was shown with each effort level, for a total of 600 trials, presented in a random order. Subjects were instructed to maintain fixation on a central fixation point; the food and effort cue always appeared to the left and right, respectively, of central fixation. After viewing each food-effort combination, subjects were asked to enter their decision as to whether they would be willing to expend the specified level of effort for the depicted item. The food-effort cue remained on the screen until the participant responded (maximum of $3.5 \mathrm{~s}$ ). Responses were entered using a four-point scale ( 1 , strong no; 2 , no; 3 , yes; 4 , strong yes), again using the left hand. This allowed us to simultaneously measure both the subjects' choices (yes/no) and the strength of their decisions (strong/weak).

Subjects were informed at the start of the decision task that their choice would determine which food and effort level they worked for on each trial. If a subject responded yes or strong yes, he would have to exert the specified level of effort for the depicted food. Otherwise, he would exert a medium amount of effort for the neutral reference food, selected on the basis of his initial liking ratings. Subjects were informed of the identity of the reference food at the start of the decision task, and it was displayed as a reminder at the beginning of each subsequent block. Thus, subjects always had to exert some level of effort on every trial, allowing us to focus in our analysis on how effort cost interacts with stimulus value to influence choice behavior. After their decision, subjects saw a preparation screen with the words "Get ready" for an average of $1000 \mathrm{~ms}$, jittered randomly between 800 and $1200 \mathrm{~ms}$.

At the end of this preparation period, the effort component commenced, with grip-strength information displayed in real time as a rising and falling red rectangle within the thermometer bar display. The display also included a picture of the food for which the effort was being expended, as well as the horizontal yellow bar signaling the threshold above which grip strength should be exerted. Subjects were instructed that the effort component of each trial would last for $2 \mathrm{~s}$. Although subjects were encouraged to exert the indicated effort for as much of the effort period as possible, because of the difficulty of maintaining a steady grip, we counted as successful any trial in which the effort exceeded the threshold on at least half of the sampled time points in the $2 \mathrm{~s}$ period. At the end of the effort period, feedback on grip performance was displayed for $500 \mathrm{~ms}$ in the form of the word "Passed!" in green or "Failed" in red. Failed trials were not repeated at any point in the experiment. Each trial was selfinitiated by the subject via pressing the space bar. During trials, subjects were instructed to maintain fixation on the central fixation point and to minimize blinks and eye movements. Adherence was monitored with the EEG equipment by checking frontal electrode measurements for stereotypical patterns of eye-movement-related artifact in the data during recording. The task was broken into three blocks of 200 trials each, with 10 min breaks between blocks.

In Part III, a single trial from the decision task was randomly selected, and subjects either did or did not receive the food from that trial, contingent on their performance in the effort period. Performance during the decision task in Part II was monitored to ensure that no more than $10 \%$ of trials constituted failures to expend the required effort. Furthermore, subjects were informed at the beginning of the decision task in Part II that whether they would receive the food at the end of the session depended on their successful exertion of effort during the trial. Together, these conditions ensured that subjects' choices mattered to them, also serving to keep the proportion of failed trials low.

EEG data acquisition and preprocessing. EEG data were collected using a 128-channel HydroCel Geodesic Sensor Net (Electrical Geodesics) with $\mathrm{AgCl}$-plated electrodes. Evoked brain potentials were digitized continuously at $500 \mathrm{~Hz}$, referenced to vertex electrode Cz. Online filtering was performed using a $200 \mathrm{~Hz}$ low-pass Bessel filter and a $0.1 \mathrm{~Hz}$ high-pass hardware filter. Impedances were kept $<50 \mathrm{k} \Omega$ and adjusted during the 10 min breaks between runs. After data collection was complete, 3D sensor positions were digitized using the Geodesic Photogrammetry System.

Offline data preprocessing was performed in MATLAB using the EEGLAB toolbox (Delorme and Makeig, 2004). Data were re-referenced to the average reference and high-pass filtered at $1 \mathrm{~Hz}$ using a two-way least-squares finite impulse response filter to remove slow voltage drifts. Analysis focused on the decision period, time-locked to the onset of the 
food-effort stimulus, as well as to the response. Epochs were extracted using a window from -200 to $2300 \mathrm{~ms}$ after stimulus onset.

Because of the unequal number of trials per condition necessitated by each subject's idiosyncratic preference data, traditional artifact rejection techniques that remove whole trials are suboptimal for our datasets. Instead, we used independent components analysis, implemented via second-order blind identification (Belouchrani et al., 1997; Tang et al., 2005), to identify and remove artifactual components without affecting the number of trials per condition. When linearly "unmixing" the EEG data into a sum of temporally correlated and spatially fixed components, this analysis can be used to separate sources of artifactual noise (e.g., eyeblinks, muscle activity, sensor noise) from meaningful cognitive activity, identified by clear stimulus- or response-locking and meaningful scalp topography (Harris et al., 2011). After classification of artifactual versus nonartifactual components, the latter can be projected back onto the scalp to produce artifact-corrected brain signals (Jung et al., 2000). From this artifact-corrected data, new stimulus-locked ( $100 \mathrm{~ms}$ before to $1000 \mathrm{~ms}$ after stimulus onset) and response-locked (600 ms before to 100 $\mathrm{ms}$ after response onset) datasets were then extracted.

EEG regression analysis. To identify sensors and time windows in which neural activity was correlated with the experimental variables of interest, EEG data for each trial and sensor were entered into a linear regression analysis. Stimulus-locked data from 100 to $1000 \mathrm{~ms}$ after stimulus onset were summed over $50 \mathrm{~ms}$ non-overlapping windows, resulting in a matrix of 128 sensors $\times 18$ time windows. Response-locked data from -600 to $90 \mathrm{~ms}$ before response onset were summed over $30 \mathrm{~ms}$ non-overlapping windows, resulting in a matrix of 128 sensors $\times 23$ time windows.

These data were entered into a linear regression model of the following form:

$$
y_{\text {sensor,time }}=\beta_{0}+\beta_{1} \text { Stimulus Value }+\beta_{2} \text { Effort Cost }+\varepsilon,
$$

where $y_{\text {sensor,time }}$ consists of trial-by-trial data (in microvolts) for a given sensor and time window, and $\beta_{0}$ is the average activity in the sensor. The Stimulus Value covariate reflects the subject's liking rating for the food presented on a given trial ( 1 for strong no to 4 for strong yes, in 0.5 units), as indicated in Part I of the experiment, whereas the Effort Cost covariate indicates the effort associated with that food ( 1 , low; 2 , medium; 3 , high). This analysis generated a map of estimated regression coefficients (i.e., $\beta$ map) for every sensor, time window, and subject. Mixed-effect group estimates were computed via one-sample $t$ tests versus zero over individual $\beta$ maps, allowing identification of which sensors and time windows show neural activity significantly correlated with stimulus value and effort cost across subjects.

Because the significance maps determined above may reflect factors epiphenomenal to choice, such as low-level visual properties or physiological arousal, we further constrained the results using a conjunction analysis with net value. A logistic function reflecting the weighted sum of stimulus value and effort cost was fit for each participant. This resulted in 21 ( 7 stimulus values $\times 3$ effort levels) unique "net value" levels assigned to the 600 trials, with the net value of each trial reflecting the combination of the individual's own liking rating and the level of effort required on that trial. An additional regression model, similar to that shown above but with only net value as a covariate, was then run to produce individual $\beta$ maps of net value, which were then aggregated into mixed-effect group estimates using a one-sample $t$ test as described above.

We assessed the conjunction of net value with effort cost and stimulus value by finding those sensors and time windows in which $p \leq 0.05$ for both net value and each covariate of interest. Because of the large number of regressions produced by each analysis (e.g., 25 subjects $\times 128$ sensors $\times 18$ time windows), all $p$ values were first corrected for multiple comparisons using an approximate permutation test, in which the labels for the 600 trials were randomly shuffled 1000 times to derive an empirical distribution of $t$ values for each sensor and time window combination. The resulting permutation-corrected $p$ values surviving the conjunction were visualized to identify sensor and time window combinations that were significantly correlated with both effort cost and stimulus value. This gives a measure of activity reflecting the construct of net value, i.e., not explained by either stimulus value or effort cost alone.
According to standard information processing models of neuroeconomic choice, the net value variable (21 conditions) should be highly correlated with decision value, or the subject's actual response (four conditions). Because the lower dimensionality of the decision value space makes for greater ease of comprehension in visual displays, we constructed average waveform displays using decision value. However, the selected sensors of interest (SOIs) were based on significant activity correlated with the net value covariate.

EEG Bayesian source reconstruction. To identify putative brain regions associated with significant EEG activity at the scalp, we used distributed source reconstructions in SPM8 (Wellcome Department of Imaging Neuroscience, Institute of Neurology, London, UK). This approach models the cortical sheet as a series of hundreds of small dipolar patches, applying an empirical Bayesian algorithm (Friston et al., 2008) that constrains the underlying sources to provide a common explanation for evoked responses across all subjects (Litvak and Friston, 2008). Unlike traditional dipole fitting, this method requires no a priori assumptions about the number or spatial locations of sources.

Here we applied a "localization of differences" approach, which assumes that there is a set of neural sources representing different levels of a psychological variable of interest (Henson et al., 2007): for example, stimulus value or effort cost. In each subject, we performed source reconstructions of the linear ordering of stimulus value, effort cost, and net value. In each subject and analysis, we computed difference waveforms by multiplying the average waveforms for each condition by the weights corresponding to a linear (monotonically increasing) trend and then summing across all weighted averages. Thus, for the three levels of effort cost (low, medium, high), we used weights of $(-1,0,+1)$; for the four levels of decision value (strong no, no, yes, strong yes), the weights would be $(-3,-1,+1,+3)$, and so on. To address the relatively large number of unique net values, we averaged net value conditions into groups of three for a total of seven conditions. Thus, both stimulus value and net value difference waveforms were computed using weights of $(-3,-2$, $-1,0,+1,+2,+3)$. For these conditions, robust averaging was used before construction of the difference waveform (Wager et al., 2005). This procedure reduces the influence of artifactual within-trial noise that may arise as a result of the relatively low number of trials per condition.

After construction of the difference waveform, subjects' data were entered into the same source space using a "canonical mesh" based on SPM's template head model, derived from the MNI brain. Individual subjects' sensor and fiducial coordinates, obtained with the Geodesic Photogrammetry System, were coregistered with the MRI coordinate system and matched to the cortical mesh via an iterative alignment algorithm. A boundary element model was used to model the source space.

Once reconstructions were complete, we identified statistically significant source estimates across individuals using $F$ tests, corrected for multiple comparisons using a stringent familywise error (FWE)corrected threshold of $p<0.01$. Results were visualized in terms of maximal intensity projection, with special focus on cortical generators because of their likely greater contribution to potentials recorded at the scalp (Cohen et al., 2011).

Granger connectivity analysis. Given the theorized role of net value signals in transforming good-based values into action selection, we were further interested in how sources associated with net value contributed to the organization of the motor response. To this end, we used Granger causality analysis via the Granger Causal Connectivity Analysis (GCCA) toolbox for MATLAB (Seth, 2010).

For this analysis, we first identified periods of significant activity in the response-locked data associated with motor output. Subjects' decision data were re-coded in terms of the finger used to make the key press (pinky, ring finger, middle finger, index finger) and entered into a linear regression with response finger as the covariate of interest. Correction for multiple comparisons was performed using permutation testing as described above. Because we wanted to focus on those time windows most strongly associated with generation of the movement-related potential, we used a significance threshold of $p=0.01$. This analysis identified two time periods of interest in which activity was strongly correlated with motor output, from -300 to $-210 \mathrm{~ms}$ and -60 to $0 \mathrm{~ms}$ before the response. 
For these two time windows of interest, we examined Granger causal connectivity in seven regions of interest (ROIs) defined from sensorimotor sources in the net value analysis (see Tables 5, 6, indicated clusters): left $\mathrm{dmFC}$ and precuneus, right premotor/motor cortex, and bilateral regions of ventrolateral PFC (vlPFC) and intraparietal sulcus (IPS).

The GCCA was performed in several steps, as described previously by Harris et al. (2013). First, we performed a Bayesian source reconstruction on the linear contrast for the motor response (pinky to index finger) over the entire time window of the response-locked data. This generated a dipole intensity map for each subject, which we then used to forward model the projected average time course for each ROI within each subject. This resulted in time series data associated with the linear ordering of the motor response for the chosen sources of interest. To satisfy the assumptions of GCCA, preprocessing steps including linear detrending, subtraction of the temporal mean, and division by the temporal SD were applied to the time course data. We then combined data across subjects into a single matrix, treating each subject as a single realization of an underlying stochastic process, followed by subtraction of the ensemble mean and division by the ensemble SD. Covariance nonstationarity was addressed by applying first-order differencing; however, augmented Dickey-Fuller and KPSS tests produced divergent results, indicating no clear evidence regarding covariance nonstationarity. Because additional differencing necessary for convergence would also complicate interpretation of the results, we considered first-order differencing sufficient to approximate the stationarity assumptions of Granger causality for our analysis.

Using the matrix of sources and data points produced by these analysis steps, we estimated the GCCA model for our two time windows of interest, from -300 to $-210 \mathrm{~ms}$ and -60 to $0 \mathrm{~ms}$ before the response. Optimal model order was selected using the Bayesian information criterion, resulting in a value of 6 (lag of $12 \mathrm{~ms}$ ). Significance was assessed using a threshold of $p=0.05$, Bonferroni corrected. For both time windows of interest, the Durbin-Watson test found no significant correlation in the residuals, and the consistency test showed high consistency of the fitted model with the correlation structure of the data $(>94 \%)$.

\section{Results}

In this experiment, we recorded behavioral responses and EEG while subjects made decisions about exerting physical effort for various appetitive foods (Fig. 1). First, subjects rated their preferences for 200 different appetitive snack foods, one at a time, repeated over two blocks to obtain average liking ratings on a four-point scale in 0.5 unit increments. For each subject, we then defined a set of neutral items (rating of 2.5), from which one option was randomly drawn to serve as the "reference food" for the following decision task. Second, subjects saw each of the same foods, paired on each trial with one of three effort levels (low, medium, high). Effort levels corresponded to 25, 50, and 75\% of the maximum grip strength measured individually for each subject, and each food was paired with all three effort levels over the course of the experiment. On each trial, subjects decided whether they wanted to exert the specified effort for the pictured food or a medium level of effort for the reference food. After their decision, subjects were required to exert the chosen level of effort for $2 \mathrm{~s}$, during which time they received real-time feedback on their grip strength. Subjects were informed at the start of the experiment that, after the task, a single trial would be randomly selected, and, depending on how they had performed in the effort period on that trial, they would or would not receive the selected food. This procedure was implemented in the third part of the experiment.

The following sections detail our analyses to investigate the role of effort cost representation in valuation and choice. First, we analyzed the behavioral data to verify that subjects took effort cost into account when making their decisions. Second, we looked at the ERP data time-locked to the onset of the food and effort cues. In line with the information processing model, stimulus-locked ERPs have been linked previously to serial stages of perception and cognition. Therefore, in our initial analysis of the stimulus-locked ERPs, we searched for brain activity correlated with net value (stimulus value - effort cost) in relation to the onset of the stimulus. Time-locking to stimulus onset also allowed us to test for the rapid emergence of effort cost signals originating from dorsal sensorimotor areas, as suggested by the affordance competition hypothesis. Third, we analyzed the same ERP data time-locked to the key-press response to assess whether and how net value signals are involved in transforming cognitive representations of value into action plans. Combining source reconstruction of response-locked data and Granger causal connectivity, we further explored how sensorimotor regions implicated in net value interact in the time leading up to preparation and execution of motor output.

\section{Behavior: effects of stimulus value and effort cost on choice}

We assessed the extent to which stimulus value and effort cost influenced subjects' choices by fitting logistic regression curves to choice (yes/no) as a function of liking rating (Fig. 2A) and effort cost (Fig. 2B). As expected, decisions were positively influenced by the subject's reported preference for the food, whereas the percentage of yes responses decreased with increasing effort levels. One-sample $t$ tests confirmed that the estimated slope terms were significantly different from zero for both liking ratings $\left(t_{(24)}=11.5, p=3.06 \times 10^{-11}\right)$ and effort cost $\left(t_{(24)}=\right.$ $\left.-6.24, p=2.0 \times 10^{-6}\right)$, indicating that both of these factors influenced subjects' decisions whether to work for particular foods versus the reference food at medium effort.

However, one potential issue is that subjects could have fundamentally switched strategies over time as a result of physical fatigue. For example, a participant could have started the experiment making decisions based exclusively on liking ratings but, as fatigue increased with physical exertion, shifted to choosing based solely on effort cost in later trials. Although we attempted to reduce this likelihood by recalibrating effort levels at the beginning of each run, decision strategy change related to effort exertion over time could potentially produce confounds in the relative timing of signals associated with stimulus value and effort cost. To evaluate this possibility, we split the 600 experimental trials into two datasets of 300 trials each, reflecting the first and second halves of the experiment, respectively. For each subject, we reran the logistic regression analysis for each dataset and compared the estimated slope terms from the first and second half for both liking ratings and effort cost. We found that the logistic slope terms associated with both factors decreased over time (Fig. $2 C)$, consistent with a reduced reliance on liking ratings and increased importance of effort level. However, for both the early and later trials, nonzero slope terms for preference (mean $\pm \mathrm{SD}$, first half, $2.16 \pm 1.07$; second half, $1.94 \pm 0.88$ ) and effort (mean $\pm \mathrm{SD}$, first half, $-1.09 \pm 0.94$; second half, $-1.27 \pm 0.99$ ) suggest that subjects were integrating both factors into their choices throughout the experiment. To verify this pattern, we entered the logistic slope terms into a $2 \times 2$ within-subjects repeated-measures ANOVA with liking/effort and first half/second half as factors; two subjects were excluded because their model fits did not converge for effort when only the first 300 trials were used. Significant main effects were found for both liking/ effort $\left(F_{(1,22)}=664.1, p=6.29 \times 10^{-18}\right)$ and first half/second half $\left(F_{(1,22)}=6.15, p=0.02\right)$. However, the interaction of these terms was not significant $(F<1)$. Therefore, despite increased sensitivity to effort cost over time, subjects do not appear to have 
fundamentally changed their strategies between the first and second halves of the experiment.

Given that both preference and effort cost appear to influence choice, an additional question is how these two factors interacted during decision-making. In particular, if preferences were consistent between the ratings session and decision blocks, we would expect higher levels of effort to be accepted for more highly rated foods. To examine this question, we computed the percentage of yes responses in the decision task for each level of effort, broken down by the seven liking rating categories. As shown in Figure 2D, subjects were much more likely to make yes responses across all levels of effort for stimuli that were rated as liked or strongly liked. Conversely, for items that were disliked or strongly disliked, subjects more rarely chose to exert the depicted level of effort, even if it was lower than that for the reference item (medium effort level). Consistent with this observation, a withinsubjects repeated-measures ANOVA with effort cost (low/medium/high) and stimulus value (strong dislike to strong like) as factors found a significant interaction of effort cost and stimulus value $\left(F_{(12,288)}=17.9\right.$, $\left.p=2.3 \times 10^{-11}\right)$, in addition to significant main effects of effort cost $\left(F_{(2,48)}=55.4, p=\right.$ $\left.5.41 \times 10^{-9}\right)$ and stimulus value $\left(F_{(6,144)}=\right.$ $\left.221.7, p=1.71 \times 10^{-25}\right)$. All reported results are adjusted for violations of sphericity using the Greenhouse-Geisser correction.

As a final check, we assessed the overall distribution of values for the decision response (Fig. 2E) and the average median response time for each response across subjects (Fig. $2 F$ ). Both of these measurements revealed an inverted $U$ shape to the response frequency and response time, with relatively fewer and faster responses in strong no and strong yes conditions. Consistent with this observation, repeatedmeasures ANOVAs with response as a factor (strong no/no/yes/ strong yes) found a significant quadratic contrast for both number of trials $\left(F_{(1,24)}=19.4, p=0.0002\right)$ and reaction time $\left(F_{(1,24)}=16.4, p=0.0005\right)$. Across all subjects and responses, the average median response time was $1.01 \mathrm{~s}$.

Stimulus-locked ERP: cognitive representation of net value According to information processing models of valuation and decision-making (Padoa-Schioppa, 2011), effort cost influences choice predominantly after the initial computation of stimulus value, when good-based representations are transformed into motor output. Consistent with this idea, signals associated with net or combined value (stimulus value - effort cost) have been localized to regions of ACC and dmFC (Croxson et al., 2009; Prévost et al., 2010; Kennerley et al., 2011; Rushworth et al., 2011; Burke et al., 2013), areas previously linked to action planning (Rushworth et al., 2007). However, given that response-related activity is often highly variable in relation to stimulus onset, it is choice. All error bars reflect SEM.

\section{B Effort Cost vs. Choice}

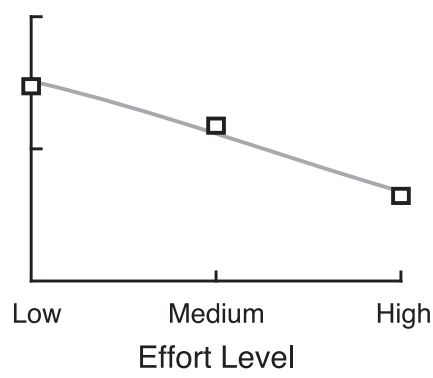

D Liking $x$ Effort

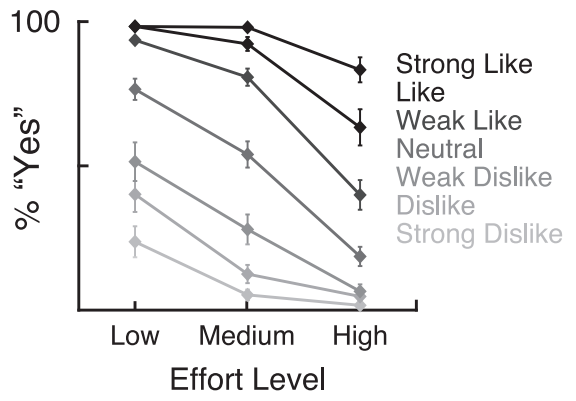

F Average Median RT

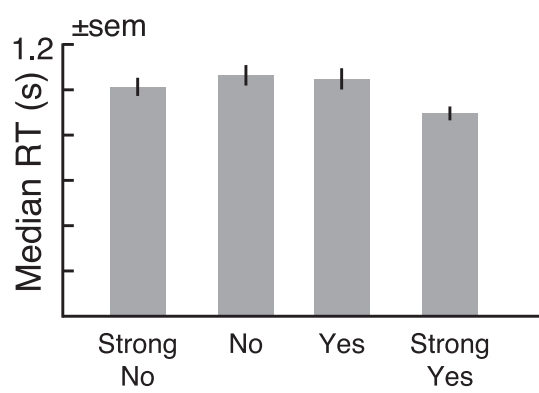

Figure 2. Behavioral results. $\boldsymbol{A}$, Group average choices as a function of liking rating. $\boldsymbol{B}$, Group average choices as a function of effort. $\boldsymbol{C}$, Logistic slope term for liking rating and effort level for first versus second half of the experiment. $\boldsymbol{D}$, Interaction of liking and effort rating in group average choices. $\boldsymbol{E}$, Histogram of responses in the decision task. $\boldsymbol{F}$, Average median response time (RT) by

less clear whether and when net value signals should emerge in relation to the onset of the stimulus.

In our first analysis, we looked for ERP responses correlated with net value, defined as stimulus value minus effort cost. For each participant, we calculated individual net value parameters by fitting the percentage of yes responses as a logistic function of the weighted sum of stimulus value and effort cost. Although net value signals have also been modeled by hyperbolic (Prévost et al., 2010) or divisive (Croxson et al., 2009) functions, neither of these options produced appreciably different model fits at the group level compared with our simpler additive approach (additive, $R^{2}=0.86$; hyperbolic, $R^{2}=0.85$; divisive, $R^{2}=0.84$ ). Figure $3 A$ displays sensor and time window combinations in which brain activity was significantly correlated with the parametric variation in net value. The $x$-axis represents time binned in $50 \mathrm{~ms}$ windows, from the earliest latencies reliably associated with visual processing ( $\sim 100 \mathrm{~ms}$ after stimulus $)$ to the range of the average median response ( $\sim 1000 \mathrm{~ms}$ after stimulus). Thus, our chosen time window focuses on the period leading up to the response, minimizing possible confounds such as visual offset transients. The $y$-axis shows sensors grouped by their position on the scalp into six 

Net Value Conjunction, Stimulus-Locked

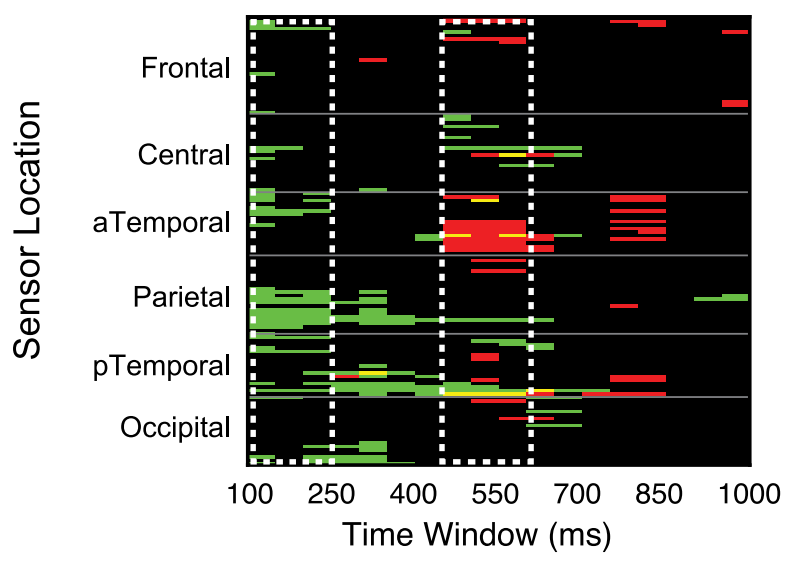

Stim Value Effort Cost Overlap

B

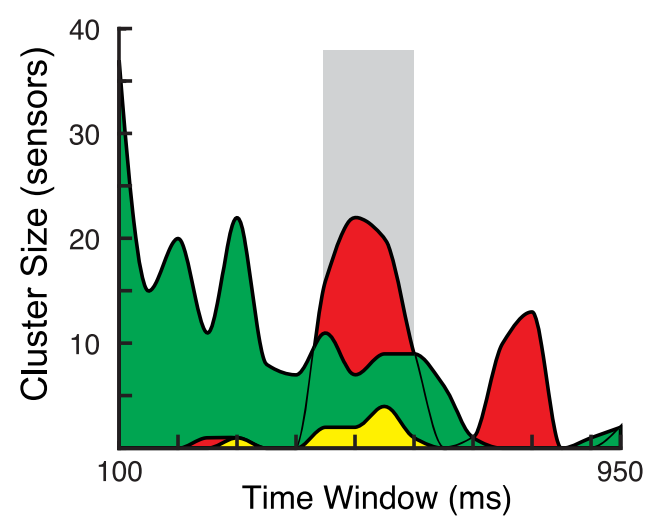

C
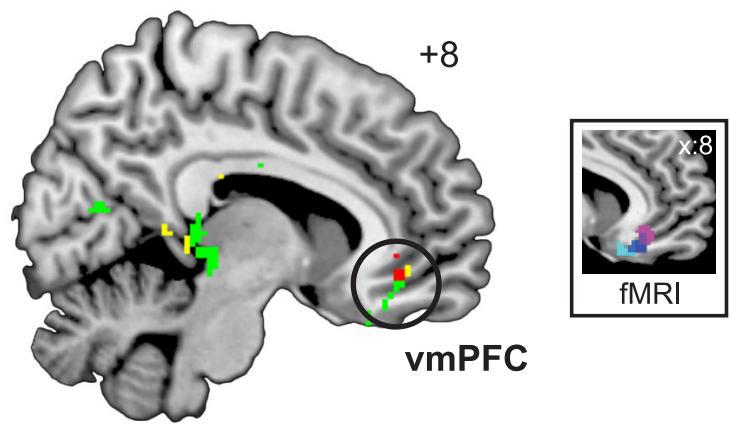

FWE $p=0.01$

Figure 3. Net value analysis, stimulus-locked data. $\boldsymbol{A}$, Heat map showing significant ERP activity correlated with net value, in conjunction with significant responses to stimulus value (red) and effort cost (green) alone. By definition, net value should reflect the overlap (yellow) of the stimulus value and effort cost activity. The data reveal an early window of activity associated with effort cost and a later window correlated with stimulus value (white dashed boxes) but relatively little overlap between the two factors. $\boldsymbol{B}$, Cluster analysis of stimulus value, effort cost, and net value. Collapsing over scalp locations, this visualization further supports the distinction between activity correlated with effort cost early (e.g., $100-250 \mathrm{~ms}$ after stimulus) and stimulus value later (450-650 ms after stimulus), while finding only a few clusters associated with the overlap of these factors in the $450-600$ ms time window (gray box). C, Source localization of net value, effort cost, and stimulus value from 450 to $600 \mathrm{~ms}$ after stimulus, FWE-corrected $p<0.01$. Inset, Spherical masks based on peak coordinates from three fMRI studies [blue, Plassmann et al. (2007); magenta, Hare et al. (2009); cyan, Litt et al. (2010)], 6 mm radius. major subdivisions: occipital, posterior temporal (pTemporal), parietal, anterior temporal (aTemporal), central, and frontal. Because high values for the net value covariate can reflect extreme levels of either stimulus value or effort cost, we applied a conjunction analysis to further quantify how much of the activity attributed to net value loaded on effort cost alone (Fig. 3A, green), stimulus value alone (Fig. $3 A$, red), or the overlap of both factors (Fig. 3A, yellow).

Because the theoretical net value computation should reflect both stimulus value and effort cost, only the overlap term constitutes a true measure of net value activity. However, within the stimulus-locked window, we found relatively few sensor-time window combinations showing significant responses to both effort cost and stimulus value. To visualize this result more clearly, we plotted the data from Figure $3 A$ in terms of the size of sensor "clusters" comprising significant activity for each time window (Fig. 3B). Although this approach removes information about where on the scalp significant activity occurred, it clearly highlights the temporal extent and number of sensors associated with each factor.

Despite the presence of large clusters of activity across the stimulus-locked window associated with effort cost (maximum cluster size, 37) and stimulus value (maximum cluster size, 22), the number of net value responses was comparatively low (maximum cluster size, 4) and concentrated in a single time period, from 450 to $600 \mathrm{~ms}$ after stimulus onset. Examining the same data with activity binned over $10 \mathrm{~ms}$ intervals revealed a similar pattern for net value from 450 to $600 \mathrm{~ms}$ (maximum cluster size, 4), despite larger clusters for effort cost (maximum cluster size, 49) and stimulus value (maximum cluster size, 26). Thus, it is unlikely that our initial use of a $50 \mathrm{~ms}$ window limited our ability to pick up potentially transient net value signals, consistent with the observation that the power of the event-related response is concentrated at lower frequencies (e.g., <20 Hz; Luck, 2005).

Localization of net value activity in this 450-600 ms window revealed sources in regions including vmPFC (Fig. 3C, left; Table 1). Although the apparent spatial precision of the sources shown here derives from a stringent statistical threshold (FWE-corrected $p<$ 0.01 ), it is notable that the vmPFC sources identified in this study overlap not only with previous source localizations (Harris et al., 2011, 2013) but also with previous results from fMRI (Fig. 3C, right; Plassmann et al., 2007; Hare et al., 2009; Litt et al., 2011). Because the coarse spatial resolution of EEG precludes precise localization of evoked potential responses, one question is whether this valuerelated activity could arise from other sources, notably ventral striatum. Functional imaging studies of reward and valuation often report coactivation of vmPFC and ventral striatum (for review, see Clithero and Rangel, 2014), although the latter appears to be particularly involved in computing reward prediction errors (Hare et al., 2008). However, the basal ganglia are located relatively deep in the brain and lack the columnar organization found in cortical pyramidal cells, making the propagation of large-amplitude potentials to the scalp less likely (Cohen et al., 2011). Therefore, given the uncertainty regarding the contribution of the basal ganglia to scalp ERPs, we have chosen to focus on putative cortical generators in this and all other source localization analyses. Despite the distance of the ventral frontal lobe from the scalp, our use of a high-density EEG array with coverage of the face and neck should maximize our ability to capture activity from sources in the basal forebrain (Holmes, 2008).

In light of the numerous studies linking vmPFC activity to subjective value computations (Kable and Glimcher, 2009; Rushworth et al., 2009), usually within a good-based framework, it is uncertain how much of this "net value" activity reflects transfor- 
Table 1. Peak MNI coordinates: net value, $450-600 \mathrm{~ms}$ after stimulus

\begin{tabular}{|c|c|c|c|c|c|c|}
\hline Voxels (n) & Side & Peak M & coordin & & $F$ & MNI coordinate region \\
\hline 459 & L & $\begin{array}{l}-30 \\
-22 \\
-12\end{array}$ & $\begin{array}{l}24 \\
26 \\
12\end{array}$ & $\begin{array}{l}-22 \\
-10 \\
-18\end{array}$ & $\begin{array}{r}158.5 \\
136.3 \\
80.0\end{array}$ & $\begin{array}{l}\text { Middle orbitofrontal cortex, } \\
\text { vmPFC }\end{array}$ \\
\hline 201 & $\mathrm{R}$ & $\begin{array}{l}24 \\
30 \\
16\end{array}$ & $\begin{array}{r}16 \\
8 \\
20\end{array}$ & $\begin{array}{l}-\mathbf{1 6} \\
-16 \\
-22\end{array}$ & $\begin{array}{r}119.8 \\
89.9 \\
85.0\end{array}$ & $\begin{array}{l}\text { vIPFC, middle orbitofrontal } \\
\text { cortex }\end{array}$ \\
\hline 63 & $\mathrm{R}$ & $\begin{array}{r}46 \\
44\end{array}$ & $\begin{array}{r}-2 \\
-12\end{array}$ & $\begin{array}{l}-14 \\
-12\end{array}$ & $\begin{array}{l}92.2 \\
78.6\end{array}$ & Insula \\
\hline 126 & L & $\begin{array}{l}-42 \\
-34 \\
-24\end{array}$ & $\begin{array}{l}-2 \\
-2 \\
-8\end{array}$ & $\begin{array}{l}-44 \\
-40 \\
-34\end{array}$ & $\begin{array}{l}85.1 \\
79.1 \\
69.2\end{array}$ & $\begin{array}{l}\text { Temporal pole, anterior } \\
\text { temporal lobe }\end{array}$ \\
\hline 72 & $\mathrm{R}$ & $\begin{array}{r}42 \\
50 \\
34\end{array}$ & $\begin{array}{l}-40 \\
-36 \\
-36\end{array}$ & $\begin{array}{l}-26 \\
-24 \\
-22\end{array}$ & $\begin{array}{l}\mathbf{8 2 . 8} \\
63.7 \\
54.9\end{array}$ & $\begin{array}{l}\text { Fusiform gyrus, inferotemporal } \\
\text { cortex }\end{array}$ \\
\hline 75 & $\mathrm{R}$ & $\begin{array}{l}18 \\
12 \\
12\end{array}$ & $\begin{array}{l}-30 \\
-36 \\
-38\end{array}$ & $\begin{array}{r}-12 \\
-2 \\
6\end{array}$ & $\begin{array}{l}\mathbf{7 2 . 3} \\
61.9 \\
61.9\end{array}$ & $\begin{array}{l}\text { Parahippocampal cortex, medial } \\
\text { temporal lobe }\end{array}$ \\
\hline 13 & $\mathrm{R}$ & 34 & -46 & 34 & 71.2 & Intraparietal cortex \\
\hline 16 & L & $\begin{array}{r}0 \\
-2\end{array}$ & $\begin{array}{l}30 \\
24\end{array}$ & $\begin{array}{r}-2 \\
12\end{array}$ & $\begin{array}{l}68.5 \\
64.9\end{array}$ & Corpus callosum \\
\hline 38 & L & $\begin{array}{l}-42 \\
-38 \\
-34\end{array}$ & $\begin{array}{r}20 \\
10 \\
-12\end{array}$ & $\begin{array}{l}12 \\
12 \\
10\end{array}$ & $\begin{array}{l}67.3 \\
62.0 \\
61.7\end{array}$ & Insula \\
\hline 6 & $\mathrm{~L}$ & -54 & -48 & 38 & 64.8 & Inferior parietal lobule \\
\hline 42 & L & -42 & -54 & 42 & 64.3 & Inferior parietal lobule \\
\hline 15 & L & -54 & -40 & -18 & 63.3 & Inferotemporal cortex \\
\hline 8 & $R$ & 46 & 18 & 6 & 63.0 & Anterior insula \\
\hline 12 & $\mathrm{~L}$ & -48 & -38 & -26 & 62.7 & Inferotemporal cortex \\
\hline 5 & L & 0 & -38 & 20 & 61.9 & Posterior cingulate cortex \\
\hline 14 & $\mathrm{R}$ & 52 & -40 & -18 & 61.0 & Fusiform gyrus \\
\hline 5 & L & -36 & -4 & -10 & 60.5 & Insula \\
\hline 14 & L & -22 & -42 & 50 & 60.4 & Somatosensory cortex \\
\hline 29 & L & $\begin{array}{l}-20 \\
-14\end{array}$ & $\begin{array}{l}-62 \\
-70\end{array}$ & $\begin{array}{l}44 \\
52\end{array}$ & $\begin{array}{r}59.5 \\
53.9\end{array}$ & Intraparietal sulcus \\
\hline 7 & $\mathrm{R}$ & 8 & 42 & -8 & 58.2 & vmPFC \\
\hline 6 & $\mathrm{R}$ & 40 & 2 & 14 & 57.1 & Insula \\
\hline 8 & $\mathrm{R}$ & 42 & -72 & -14 & 55.1 & Inferior occipital cortex \\
\hline 7 & $\mathrm{R}$ & 40 & -20 & -32 & 54.2 & Inferotemporal cortex \\
\hline 5 & $\mathrm{R}$ & 24 & -38 & -10 & 54.0 & Parahippocampus cortex \\
\hline 11 & L & -10 & -90 & -18 & 53.9 & Occipital pole \\
\hline 5 & $\mathrm{R}$ & 36 & -70 & 28 & 53.5 & Temporoparietal cortex \\
\hline 6 & L & $\begin{array}{l}-16 \\
-16\end{array}$ & $\begin{array}{l}-46 \\
-36\end{array}$ & $\begin{array}{r}-6 \\
-10\end{array}$ & $\begin{array}{l}52.6 \\
52.5\end{array}$ & $\begin{array}{l}\text { Parahippocampal gyrus } \\
\text { Medial temporal lobe }\end{array}$ \\
\hline
\end{tabular}

Clusters surviving FWE-corrected threshold $p<0.01(F=50.6)$ and cluster size threshold $k=5$. L, Left; $\mathrm{R}$, right. In this and all other tables, bold indicates a cluster-level maximum, with separate $(>8 \mathrm{~mm})$ maxima listed in plain type below. Due to the low spatial resolution of $\mathrm{EEG}$, source regions reflect the general location of source activity rather than precise coordinates.

mation of effort cost into motor output, as proposed in the information processing framework, versus the valuation of the effort cost cue itself and/or related haptic information. Consistent with the latter idea, both stimulus value and effort cost signals within this time window were also localized to vmPFC, whereas we failed to observe net value activity localized to previously reported regions of ACC and dmFC (Croxson et al., 2009; Prévost et al., 2010).

\section{Stimulus-locked ERP: early sensorimotor representation of effort cost}

Our next ERP analysis looked more specifically at the emergence of neural activity correlated with effort cost and stimulus value after the onset of the combined food-effort cue. In particular, based on previous data, we hypothesized that sensorimotor regions would be involved in representing effort cost from rela- tively early in the trial (Cisek and Kalaska, 2010), whereas signals associated with stimulus value would occur later, from $\sim 450$ to $650 \mathrm{~ms}$ after stimulus onset, localized to regions including vmPFC (Harris et al., 2011, 2013).

Re-examining the cluster analysis in Figure $3 B$ revealed that brain activity reflected both stimulus value and effort in the time leading up to choice. Yet these mental operations did not unfold simultaneously but rather showed a striking temporal sequence. Although effort cost computations occurred across the time course of choice, cluster sizes were largest in the first $300 \mathrm{~ms}$ after stimulus onset (Fig. 3B, green). In contrast, activity associated with stimulus value (Fig. $3 B$, red) was clustered in two major time windows, from 450 to $600 \mathrm{~ms}$ and 750 to $850 \mathrm{~ms}$ after stimulus.

We further quantified these effects by examining the relationship between the scalp topography associated with net value and those for effort cost and stimulus value. For SOIs defined as showing significant activity correlated with net value, we focused on two periods of high sensor clustering visible in the heat-map data (Fig. 3A, white boxes): 100 to $250 \mathrm{~ms}$, commonly linked to early perceptual processing, and 450 to $600 \mathrm{~ms}$, within our a priori window for value computation. During both of these periods, sensors identified on the basis of net value activity showed responses highly consistent with the eventual decision value ranging from strong no to strong yes (Fig. $4 A, B$ ), as expected given the strong relationship between net value and choice data seen in the behavioral responses. However, in line with the conjunction analysis above, examination of the scalp topography for stimulus value versus effort cost suggested differential contributions of the latter two factors to the net value signal.

At $100 \mathrm{~ms}$ after stimulus onset, net value activity was strongly lateralized over posterior left hemisphere sensors (Fig. 4A, left). Scalp topography for effort cost (Fig. $4 A$, top right) was highly similar but with reversed polarity, matching the negative effect of high effort cost on valuation in the behavioral data. In contrast, the scalp topography for stimulus value at $100 \mathrm{~ms}$ was nonsignificant (Fig. $4 A$, bottom right). Given that this early latency is traditionally associated with early sensory processing, one possibility is that net value activity in this window reflects perceptual processing of the effort cue, which was always presented on the contralateral (right) side of the screen. Alternatively, in line with the idea of rapid sensorimotor activity, this pattern could reflect somatosensory feedback and/or rapid action planning in somatosensory and motor cortices contralateral to the right hand, which held the grip dynamometer. These points are discussed further below in relation to the results of Bayesian source reconstruction of the early effort cost signal.

In the later time window, from 450 to $600 \mathrm{~ms}$ after stimulus, maps of the net value response indicate a continued contribution of effort cost, as well as the emergence of activity associated with stimulus value (Fig. 4B). As described above, net value activity during this period was localized to regions including vmPFC, in line with previous reports of value computation in this time window (Harris et al., 2011, 2013). This convergence of responses to different types of cues, food stimulus and effort level, supports the idea that vmPFC integrates information from multiple sources to produce a subjective value signal for use in decision-making.

Although these data suggest that effort cost is processed earlier than stimulus value, one potential confound in our experimental paradigm is the relative complexity of the visual stimuli associated with each factor. Whereas the computation of stimulus value required the recognition of food photographs, the effort cue was much simpler and visually more straightforward: the higher the 
A

Net Value Activity, 100-250 ms Post-Stimulus

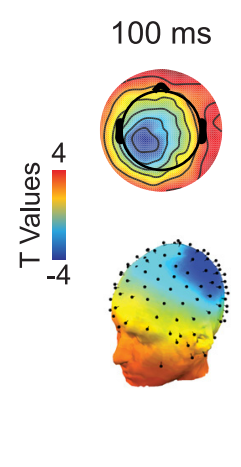

Effort Cost

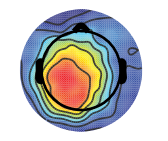

Stim Value

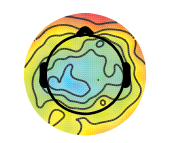

B

Net Value Activity, 450-600 ms Post-Stimulus
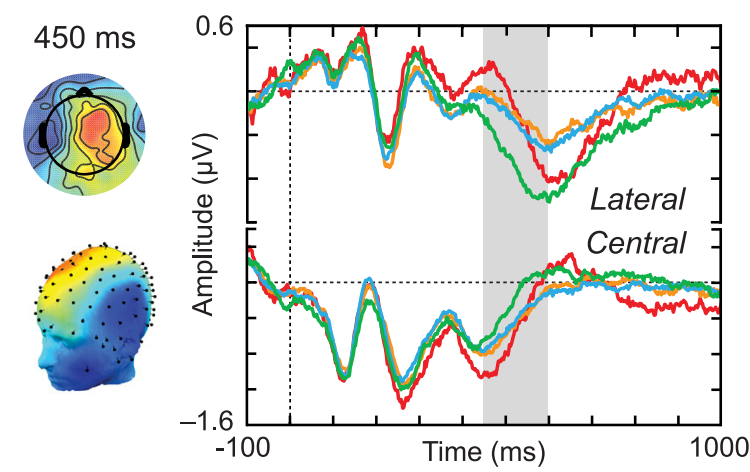

Effort Cost

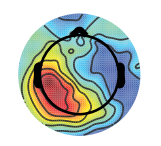

Stim Value
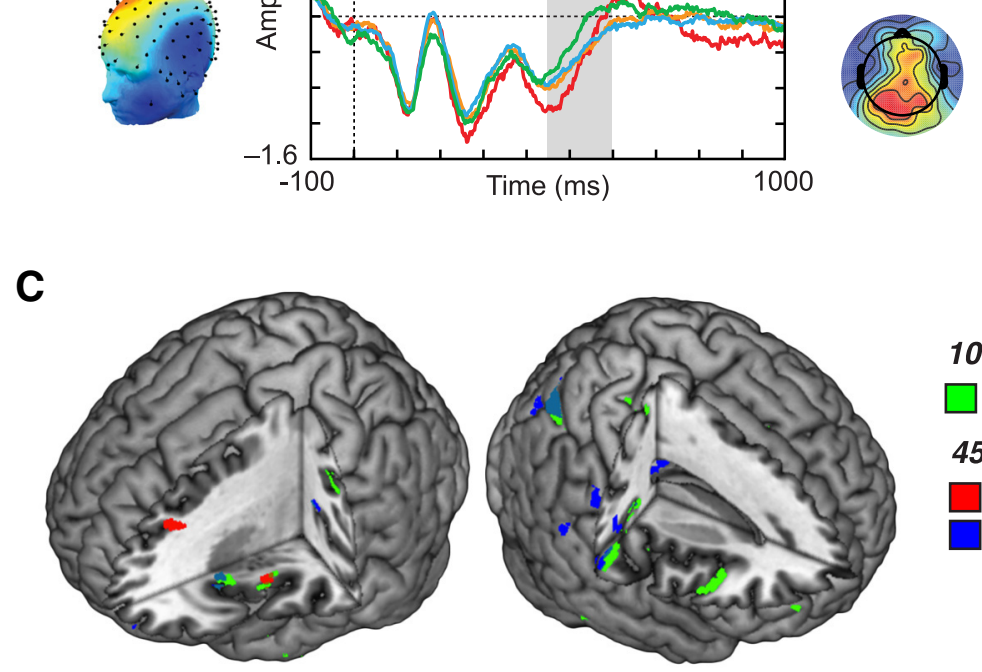

FWE $p=0.01$

Figure 4. Net value scalp topography and average waveforms, stimulus-locked data. For ease of visualization, waveforms for decision value (strong no to strong yes) are depicted for Sols selected from the net value comparison. $A$, Net value activity, $100-250 \mathrm{~ms}$ after stimulus. In line with the cluster analysis, scalp topography in this window appears to predominantly reflect effort cost (top right) rather than stimulus value (bottom right). $\boldsymbol{B}$, Net value activity, $450-600 \mathrm{~ms}$ after stimulus. In this window, both effort cost and stimulus value appear to contribute to the topography of the net value response. C, Source localization of effort cost and stimulus value, FWE-corrected $p<0.01$.

bar, the larger the effort. Thus, effort cost could be computed more quickly simply because visual processing of this stimulus had already finished while recognition of the food stimulus was still ongoing. In this case, the early neural activity associated with effort cost would merely reflect a faster visual evoked potential for the effort cue relative to the food stimulus. This is particularly problematic because the timing of the effort cost response (100$300 \mathrm{~ms}$ after stimulus onset) overlaps with the known latencies of the P1, N1, and P2 components, a series of deflections that are associated with visual perception (Luck, 2005). Peaking at $100-$ $130,150-200$, and $200-250 \mathrm{~ms}$, respectively, after stimulus onset, the P1, N1, and P2 are thought to reflect a hierarchy of visual perception from basic sensory processing to high-level object rec-

Strong

YES

$\square$

Strong

NO
100-250 ms $\square$ Effort Cost

450-600 ms

Stim Value

Effort Cost

ognition (Luck, 2005), originating in occipitotemporal regions of visual extrastriate cortex (Di Russo et al., 2002). Although most investigations of these components have focused on amplitude measures, there is suggestive evidence that their latencies can also be modulated by task demands (Taylor, 2002; Fort et al., 2005).

To examine this alternative, we measured the latency of the P1-N1-P2 complex for the effort and stimulus cues. First, we identified the P1-N1-P2 complex by taking the grand average waveform across all occipital sensors and subjects. Based on this waveform, we defined latency ranges for each component (P1, 80-150 ms; N1, 150-200 ms; P2, 200-250 ms), which were searched using the MATLAB max $(\mathrm{P} 1, \mathrm{P} 2)$ and $\min (\mathrm{N} 1)$ functions to obtain individual peak latencies in each subject. Peak latencies were acquired separately for three levels of effort cost (low, medium, high) and stimulus value (dislike, neutral, like), in the latter case collapsing across strong and weak ratings within each preference valence. These numbers were then entered into a repeatedmeasures ANOVA with condition (2, effort cost/stimulus value), level (3, low/ medium/high), and component (3, P1/ $\mathrm{N} 1 / \mathrm{P} 2$ ) as factors. As expected, the main effect of component was highly significant $\left(F_{(2,48)}=736.8, p=9.43 \times 10^{-37}\right)$, given the different peak latencies of the three components (mean $\pm \mathrm{SD}, \mathrm{P} 1,116.6 \pm$ $15.8 \mathrm{~ms} ; \mathrm{N} 1,171.3 \pm 10.5 \mathrm{~ms} ; \mathrm{P} 2,239.4 \pm$ $15.9 \mathrm{~ms}$ ). Additionally, there was a significant interaction of condition $\times$ level $\left(F_{(2,48)}=5.04, p=0.02\right)$, reflecting the fact that the medium effort cost cue was processed more quickly than the neutral stimulus value, whereas the opposite was true for low levels of effort cost and stimulus value. Critically, however, both the main effect of condition and all interactions of condition and component were not significant (all $F$ values $<1$ ). Although the indirect relationship between neural sources and surface electrodes limits our inferences regarding the underlying cortical generators of these effects, these results nonetheless provide suggestive evidence that visual processing of the food stimulus is not delayed relative to the effort cost cue.

Localization of activity attributed separately to stimulus value and effort cost revealed a number of regions linked to various cognitive processes (Fig. 4C; Tables 2-4). Most noteworthy from the perspective of the affordance competition hypothesis, effort cost activity across the time course of decision was localized to regions associated with sensorimotor processing. These included traditional sensorimotor regions, such as motor and somatosensory cortices, as well as parietal areas associated with visuomotor integration and action planning. Many of these sources were ac- 
Table 2. Peak MNI coordinates: effort cost, $\mathbf{1 0 0}-\mathbf{2 5 0} \mathrm{ms}$ after stimulus

\begin{tabular}{|c|c|c|c|c|c|c|}
\hline \multirow{2}{*}{$\frac{\text { Voxels }(n)}{136}$} & \multirow{2}{*}{ Side } & \multicolumn{3}{|c|}{ Peak MNI coordinates } & \multirow{2}{*}{$\begin{array}{l}F \\
149.9\end{array}$} & \multirow{2}{*}{$\frac{\text { MNI coordinate region }}{\text { Medial orbitofrontal cortex }}$} \\
\hline & & -8 & 6 & -16 & & \\
\hline & & -10 & 14 & -18 & 108.3 & \\
\hline & & -2 & 6 & 12 & 65.9 & \\
\hline \multirow[t]{3}{*}{36} & $\mathrm{~L}$ & -40 & 20 & 2 & 93.9 & Insula \\
\hline & & -32 & 18 & 10 & 61.2 & \\
\hline & & -28 & 10 & 14 & 51.4 & \\
\hline \multirow[t]{2}{*}{63} & L & -20 & 36 & -20 & 92.5 & Middle orbitofrontal \\
\hline & & -32 & 22 & -22 & 51.5 & cortex \\
\hline \multirow[t]{2}{*}{78} & $\mathrm{R}$ & 14 & -38 & -20 & 91.6 & Middle cingulate cortex \\
\hline & & 16 & -34 & -22 & 87.0 & \\
\hline \multirow[t]{3}{*}{232} & L & -40 & 2 & -4 & 85.3 & Insula temporal pole \\
\hline & & -28 & 16 & -10 & 76.8 & \\
\hline & & -48 & -2 & -44 & 70.2 & \\
\hline 5 & L & -52 & -14 & -46 & 82.0 & Motor cortex \\
\hline 17 & $\mathrm{R}$ & 12 & 22 & -14 & 77.9 & Orbitofrontal cortex \\
\hline \multirow[t]{3}{*}{210} & L & -12 & -28 & 34 & 77.7 & Posterior cingulate cortex \\
\hline & & -4 & -36 & 38 & 73.5 & \\
\hline & & -10 & -30 & 46 & 67.1 & \\
\hline \multirow[t]{3}{*}{104} & $\mathrm{R}$ & 48 & -18 & -16 & 77.2 & Middle temporal gyrus \\
\hline & & 48 & -6 & -22 & 63.9 & \\
\hline & & 54 & -28 & -2 & 67.1 & \\
\hline 5 & $\mathrm{R}$ & 6 & -6 & 32 & 75.9 & Middle cingulate cortex \\
\hline 58 & L & -52 & -18 & -16 & 75.9 & Middle temporal lobe \\
\hline 62 & L & -36 & 44 & 10 & 75.5 & $\begin{array}{l}\text { Dorsolateral prefrontal } \\
\text { cortex }\end{array}$ \\
\hline 12 & $\mathrm{~L}$ & -60 & -12 & 24 & 75.4 & Somatosensory cortex \\
\hline 44 & $\mathrm{R}$ & 38 & -12 & -4 & 75.4 & Insula \\
\hline \multirow[t]{2}{*}{128} & $\mathrm{R}$ & 32 & -56 & 52 & 74.4 & Superior parietal lobule, \\
\hline & & 24 & -66 & 58 & 72.6 & IPS \\
\hline \multirow[t]{2}{*}{28} & R & 50 & -14 & 16 & 73.8 & Secondary somatosensory \\
\hline & & 60 & -10 & 22 & 65.5 & cortex \\
\hline \multirow[t]{3}{*}{65} & $\mathrm{R}$ & 32 & -36 & -6 & 67.5 & Hippocampus \\
\hline & & 26 & -34 & -12 & 61.3 & Parahippocampal cortex \\
\hline & & 8 & -32 & 6 & 52.7 & Retrosplenial cortex \\
\hline 8 & L & -12 & -4 & 44 & 65.1 & Middle cingulate cortex \\
\hline \multirow[t]{3}{*}{52} & $\mathrm{R}$ & 30 & -24 & 18 & 62.8 & Posterior insula \\
\hline & & 42 & -28 & 6 & 62.7 & \\
\hline & & 38 & -28 & 14 & 62.6 & \\
\hline 43 & $\mathrm{~L}$ & -50 & -34 & -28 & 61.4 & Inferotemporal cortex \\
\hline 6 & L & -8 & 22 & 22 & 61.3 & Anterior cingulate cortex \\
\hline 7 & L & -52 & -22 & 44 & 61.0 & Somatosensory cortex \\
\hline 7 & $\mathrm{R}$ & 18 & -36 & 60 & 59.8 & Somatosensory cortex \\
\hline \multirow[t]{2}{*}{8} & L & -38 & -32 & 38 & 59.5 & Somatosensory cortex \\
\hline & & -42 & -28 & 44 & 59.0 & \\
\hline 13 & $R$ & 20 & -30 & 58 & 59.1 & Motor cortex \\
\hline 8 & $\mathrm{R}$ & 26 & 16 & 38 & 58.4 & Middle frontal gyrus/BA 8 \\
\hline 5 & $\mathrm{R}$ & 26 & -4 & 56 & 58.2 & Premotor cortex \\
\hline \multirow[t]{2}{*}{27} & $\mathrm{R}$ & 40 & 20 & 10 & 58.2 & Insula \\
\hline & & 52 & 22 & 8 & 54.2 & Inferior frontal gyrus \\
\hline 7 & L & -42 & -46 & -22 & 54.5 & Fusiform gyrus \\
\hline 11 & L & -10 & 54 & 30 & 54.4 & Superior frontal gyrus \\
\hline \multirow[t]{2}{*}{6} & L & -32 & 0 & 8 & 53.4 & Insula \\
\hline & & -30 & 8 & 12 & 52.7 & \\
\hline 10 & $\mathrm{R}$ & 62 & -32 & -28 & 53.3 & Inferotemporal cortex \\
\hline 15 & L & 0 & 28 & 0 & 52.4 & Corpus callosum \\
\hline & & 0 & 24 & 8 & 52.3 & \\
\hline
\end{tabular}

Clusters surviving FWE-corrected threshold $p<0.01(F=49.2)$ and cluster size threshold $k=5$. L, Left; $R$, right.

tive from the earliest window of activity, 100 to $250 \mathrm{~ms}$ after stimulus onset (Table 2). As mentioned above, this time window is usually associated with perceptual processing, and, indeed, sources in this period included regions of inferotemporal cortex linked to visual object recognition. However, the numerous additional sources in sensorimotor areas are not consistent with a purely perceptual stage, as hypothesized in the information pro-
Table 3. Peak MNI coordinates: stimulus value, 450 to $600 \mathrm{~ms}$ after stimulus

\begin{tabular}{|c|c|c|c|c|c|c|}
\hline \multirow{2}{*}{$\begin{array}{l}\text { Voxels }(n) \\
137\end{array}$} & \multirow{2}{*}{$\begin{array}{l}\text { Side } \\
\mathrm{L}\end{array}$} & \multicolumn{3}{|c|}{ Peak MNI coordinates } & \multirow{2}{*}{$\begin{array}{l}F \\
127.4\end{array}$} & \multirow{2}{*}{$\begin{array}{l}\text { MNI coordinate region } \\
\text { Middle orbitofrontal gyrus }\end{array}$} \\
\hline & & -32 & 22 & -22 & & \\
\hline & & -20 & 32 & -20 & 110.5 & Insula \\
\hline & & -24 & 26 & -16 & 98.3 & \\
\hline \multirow[t]{3}{*}{80} & $\mathrm{R}$ & 28 & 12 & -14 & 105.2 & Inferior frontal gyrus \\
\hline & & 18 & 26 & -26 & 95.8 & Middle orbitofrontal gyrus \\
\hline & & 40 & -4 & -16 & 69.4 & Insula \\
\hline 23 & $\mathrm{~L}$ & -22 & 36 & 30 & 71.2 & Superior frontal gyrus \\
\hline 11 & $\mathrm{R}$ & 10 & 38 & -6 & 63.2 & Ventromedial prefrontal cortex \\
\hline \multirow[t]{3}{*}{50} & L & -32 & -10 & -30 & 57.6 & Medial temporal lobe \\
\hline & & -28 & -4 & -40 & 56.6 & Temporal pole \\
\hline & & -24 & -8 & -34 & 56.5 & \\
\hline 6 & $\mathrm{~L}$ & -38 & 44 & 10 & 56.9 & Dorsolateral prefrontal cortex \\
\hline \multirow[t]{2}{*}{13} & $\mathrm{R}$ & 24 & 0 & -36 & 56.6 & Medial temporal lobe \\
\hline & & 26 & 0 & -28 & 51.7 & \\
\hline
\end{tabular}

Clusters surviving FWE-corrected threshold $p<0.01(F=49.1)$ and cluster size threshold $k=5$. L, Left; $R$, right.

cessing model. Instead, these data support the rapid feedforward processing of relevant action information theorized by models of parallel sensory and motor architecture, at least when the cost of physical effort is highly salient to the decision process.

One potential concern is that this activity could represent a conscious response to the demands of the experiment, with subjects imagining the required effort level at the onset of the trial. Given the repeated observation that motor imagery activates the same sensorimotor network involved in overt action (for review, see Jeannerod, 2001), this issue cannot be ruled out. However, one factor that makes this unlikely is the early latency of effort cost processing, preceding components previously associated with conscious motor preparation (Kranczioch et al., 2009). Although the rapid encoding of effort cost found here may reflect motor preparation or prospective action judgment, this process is probably primarily nonconscious in nature.

This pattern of localization to sensorimotor regions was not visible for the stimulus value covariate, which emerged comparatively later in the decision process and mostly reflected sources associated with executive function, such as dorsolateral PFC and vlPFC (Table 3). Extending from regions of orbitofrontal cortex into insula, the latter cluster overlapped with similar sources in both time windows of the effort cost analysis, suggesting a common locus of decision-making activity across the time course of choice. Similarly, signals for both effort cost and stimulus value were localized to regions of insular cortex across both time windows of interest, in line with the role of this brain structure in interoceptive processes, including pain, gustation, and sensorimotor coordination (Craig, 2002).

Response-locked ERP: representation of net value in dmFC The above results support the early involvement of sensorimotor networks in representing information about physical effort, with effort cost computations emerging as early as $100-150 \mathrm{~ms}$ after stimulus onset. However, we found substantially less evidence for a net value signal reflecting the transformation of stimulus values into action space, a role in which $\mathrm{dmFC}$ has previously been implicated. Because motor responses tend to be variable with respect to the stimulus onset, a better analysis approach is to examine activity time-locked to the motor response itself: in this case, the decision whether to exert the specified amount of effort for the depicted food. Because subjects held the grip dynamometer in their dominant (right) hand, this decision was indicated with a key press using the nondominant hand. Key-press responses were entered on a standard USB keyboard (Dell Com- 
Table 4. Peak MNI coordinates: effort cost, $\mathbf{4 5 0}$ - $600 \mathrm{~ms}$ after stimulus

\begin{tabular}{|c|c|c|c|c|c|c|}
\hline \multirow{2}{*}{$\begin{array}{l}\text { Voxels }(n) \\
163\end{array}$} & \multirow{2}{*}{$\begin{array}{l}\text { Side } \\
L\end{array}$} & \multicolumn{3}{|c|}{ Peak MNI coordinates } & \multirow{2}{*}{$\begin{array}{l}F \\
120.0\end{array}$} & \multirow{2}{*}{$\begin{array}{l}\text { MNI coordinate region } \\
\text { Insula, dorsolateral prefrontal }\end{array}$} \\
\hline & & -40 & 20 & 2 & & \\
\hline & & -36 & 46 & 10 & 80.2 & cortex \\
\hline & & -36 & 36 & 6 & 69.5 & \\
\hline \multirow[t]{3}{*}{150} & $\mathrm{R}$ & 30 & -36 & -8 & 115.8 & Hippocampus \\
\hline & & 8 & -34 & 6 & 79.8 & Retrosplenial cortex \\
\hline & & 22 & -32 & -6 & 75.1 & Parahippocampal cortex \\
\hline \multirow[t]{3}{*}{301} & $\mathrm{~L}$ & -20 & 38 & -18 & 112.5 & Middle orbitofrontal cortex, \\
\hline & & -6 & 6 & -8 & 78.8 & vmPFC \\
\hline & & -10 & 18 & -20 & 78.3 & \\
\hline \multirow[t]{3}{*}{68} & $\mathrm{R}$ & 12 & 26 & -16 & 106.7 & vmPFC, middle orbitofrontal \\
\hline & & 30 & 4 & -14 & 62.1 & cortex \\
\hline & & 18 & 32 & -22 & 61.1 & \\
\hline \multirow[t]{2}{*}{28} & $\mathrm{R}$ & 48 & -14 & 18 & 75.5 & Secondary somatosensory \\
\hline & & 50 & -28 & 24 & 58.0 & cortex \\
\hline \multirow[t]{2}{*}{18} & $\mathrm{~L}$ & -32 & -26 & 18 & 73.2 & Posterior insula \\
\hline & & -44 & -18 & 20 & & Somatosensory cortex \\
\hline 10 & $\mathrm{R}$ & 4 & -12 & 26 & 73.0 & Midcingulate cortex \\
\hline 9 & $\mathrm{R}$ & 52 & -50 & 24 & 72.4 & Temporoparietal junction \\
\hline \multirow[t]{3}{*}{111} & $\mathrm{R}$ & 34 & 38 & 24 & 72.3 & Dorsolateral prefrontal \\
\hline & & 40 & 34 & 34 & 60.8 & cortex \\
\hline & & 48 & 14 & 26 & 54.0 & \\
\hline 42 & $\mathrm{R}$ & 16 & -72 & 52 & 71.8 & Superior parietal lobule \\
\hline \multirow[t]{2}{*}{16} & $\mathrm{~L}$ & -60 & -8 & 10 & 71.7 & Somatosensory cortex \\
\hline & & -50 & -12 & 18 & 71.3 & \\
\hline \multirow[t]{2}{*}{57} & $\mathrm{R}$ & 32 & -62 & 56 & 69.3 & IPS \\
\hline & & 22 & -66 & 60 & 63.8 & \\
\hline 48 & $\mathrm{~L}$ & -2 & -80 & 28 & 67.4 & Occipital cortex \\
\hline \multirow[t]{2}{*}{8} & $\mathrm{R}$ & 32 & -22 & 20 & 66.8 & Insula \\
\hline & & 40 & -18 & 18 & 61.1 & \\
\hline 8 & $\mathrm{~L}$ & -52 & -48 & 20 & 65.9 & Superior temporal cortex \\
\hline 8 & $L$ & -38 & -16 & -18 & 64.8 & Medial temporal lobe \\
\hline 9 & $\mathrm{~L}$ & -46 & -42 & 34 & 64.5 & Supramarginal gyrus \\
\hline \multirow[t]{3}{*}{123} & $\mathrm{~L}$ & -14 & -38 & 52 & 64.4 & Posterior cingulate cortex, \\
\hline & & -6 & -40 & 44 & 56.1 & precuneus \\
\hline & & -8 & -42 & 60 & 51.8 & \\
\hline 15 & $\mathrm{R}$ & 8 & -66 & 14 & 63.2 & Occipital cortex \\
\hline \multirow[t]{3}{*}{72} & $\mathrm{~L}$ & -22 & -66 & 36 & 62.5 & IPS \\
\hline & & -26 & -66 & 28 & 59.8 & \\
\hline & & -14 & -72 & 46 & 52.1 & \\
\hline \multirow[t]{3}{*}{30} & $\mathrm{R}$ & 8 & 42 & -8 & 61.8 & vmPFC \\
\hline & & 8 & 34 & -18 & 54.0 & \\
\hline & & 4 & 30 & -24 & 53.7 & \\
\hline 16 & $\mathrm{R}$ & 62 & -24 & 10 & 60.8 & Superior temporal sulcus \\
\hline 6 & $\mathrm{~L}$ & -48 & -4 & -44 & 59.8 & Temporal pole \\
\hline 12 & $L$ & -48 & 2 & -32 & 56.2 & Temporal pole \\
\hline 14 & $\mathrm{R}$ & 58 & -30 & 42 & 55.3 & Supramarginal gyrus/BA 2 \\
\hline 15 & $\mathrm{~L}$ & -50 & -30 & -28 & 55.1 & Inferotemporal cortex \\
\hline 9 & $\mathrm{R}$ & 20 & -66 & 30 & 52.4 & Precuneus \\
\hline
\end{tabular}

Clusters surviving FWE-corrected threshold $p<0.01(F=49.9)$ and cluster size threshold $k=5$. L, Left; R, right.

puters); because the latency of keyboards is slow and variable, we could not precisely estimate the time of the response. However, the additional noise introduced by this imprecision should, if anything, reduce our likelihood of finding significant neural activity time-locked to the response. Figure $5 A$ displays responselocked ERP signals surviving the conjunction of net value with stimulus value (red) and effort cost (green). As detailed above, net value is defined as the combination of stimulus value with effort cost; therefore, only those sensors showing overlapping activity for these two factors (yellow) are thought to reflect true net value computations. From this analysis, we identified two major windows associated with net value activity: -420 to -390 $\mathrm{ms}$ and -60 to $0 \mathrm{~ms}$ before response (Fig. $5 A$, white dashed boxes). Viewing the same data in terms of sensor clusters reach- ing significance (Fig. 5B) reveals a similar pattern: although the highest levels of activity overall are associated with stimulus value (maximum cluster size, 35), there are two windows of peak activity for both effort cost (maximum cluster size, 12) and net value (maximum cluster size, 10), corresponding to the -420 to -390 $\mathrm{ms}$ and -60 to $0 \mathrm{~ms}$ pre-response time bins.

Looking at the corresponding scalp maps in the -420 to -390 ms period, the topography of the net value response in this window (Fig. 5C, left) is consistent with both effort cost (Fig. 5C, top right), again with inverted polarity, and stimulus value activity (Fig. 5C, bottom right). These observations further support the idea that the net value signal in this window reflects an integration of stimulus value and effort cost activity.

We also examined the average waveform across SOIs defined by significant activity correlated with net value in this time period (Fig. $5 C$, center). Unsurprisingly, as in the stimulus-locked data, we found a linear ordering of the ERP response consistent with the later decision value. Furthermore, at the same SOIs, we observed a prominent later deflection peaking at $\sim 60 \mathrm{~ms}$ before the key-press response, consistent with previous reports of movement-related activity from both EEG and subdural electrodes (Toro et al., 1994; Urbano et al., 1998). The amplitude of this motor potential showed a clear ordering from strong no to strong yes, suggesting that these sensors did indeed register activity associated with the button-press response.

Given that the response mapping was counterbalanced across subjects, this finding was unexpected, because the same decision should be associated with different motor output across subjects. For example, strong no corresponds to a flexion of the pinky finger in some subjects but an index finger movement in others. Therefore, the motor potentials associated with each group should average out, at least in theory. However, additional investigation revealed that, although some movement-related activity could be observed for both groups of subjects, one group had a much larger percentage of individuals showing a strong evoked motor potential. These subjects' strong movement-related signals thus contributed more dramatically to the average activity from -60 to $0 \mathrm{~ms}$ before response, producing a strong net value correlated response in this time window immediately preceding response. Because the size of the motor potential reflects a number of uncontrolled factors, including the exact placement of parietal electrodes and the anatomical folding and functional organization of the cerebral cortex, the finding of a motor potential in the net value contrast was completely unforeseen. Nonetheless, as discussed below, it provides a serendipitous opportunity to use Granger causal connectivity to study how brain regions associated with net value activity interact in the time leading up to the motor response. Note that this asymmetry between groups in the motor potential did not appear to influence the earlier net value signal from -420 to $-390 \mathrm{~ms}$ : we found similar polarity and topography of the net value response for the two groups in this time window, in contrast to the later period associated with the evoked motor potential. Thus, our net value effect does not appear to be merely capturing motor preparation in a subset of subjects but rather a stage of good-to-action transformation before motor effector specification. Figure $6 \mathrm{~A}$ shows the source reconstruction of net value signals from -420 to $-390 \mathrm{~ms}$ (yellow) and -60 to $0 \mathrm{~ms}$ (magenta) before response. Based on previous findings from the electrophysiological and neuroimaging literature, we had predicted that net value signals would be localized to portions of ACC and/or dmFC (Croxson et al., 2009; Prévost et al., 2010; Kennerley et al., 2011; Rushworth et al., 2011; Burke et al., 2013). Known for their connections to both decision-making and motor control regions (Paus, 2001), these areas have been theorized to play a role in inte- 
grating good-based value with action selection to produce net value signals (PadoaSchioppa, 2011). In line with this idea, distributed reconstructions of net value activity from -420 to $-390 \mathrm{~ms}$ in the response-locked data identified a cluster of significant sources spanning anterior to middle cingulate cortex, along with dmFC (Fig. 6A, circled). Despite the coarse spatial resolution of EEG, these sources lie in close proximity to activations correlated with net value in previous neuroimaging studies (Fig. 6A, inset).

Source reconstructions in the -420 to -390 ms window also highlighted a number of regions associated with action selection and motor output (Fig. 6A; Table 5). Sources in vlPFC fit with the putative role of this region in adjusting responses to reflect the current behavioral context (Christoff et al., 2001; Ramnani and Owen, 2004; Koechlin and Hyafil, 2007; Hampshire et al., 2010; Mitchell, 2011). Localization of activity to IPS and somatosensory cortex, regions implicated in action planning and execution (Creem and Proffitt, 2001; Dijkerman and de Haan, 2007), included portions of these structures previously linked to representation of the arm and hand, as corroborated by automated meta-analysis (Yarkoni et al., 2011). Finally, extensive sources in the precuneus, particularly in the left hemisphere, may reflect functional networks including an anterior sensorimotor circuit connecting to premotor and parietal cortices and/or a midcentral "cognitive/associative" network with projections to PFC (Margulies et al., 2009).

We were also able to examine the origins of the motor signal within the net value dataset (Fig. 6A, magenta; Table 6). As described in Materials and Methods, we counterbalanced response mappings between subjects in our experimental design such that, in the net value comparison, motor responses should have averaged out across participants. However, because one of the counterbalanced groups contained many more individuals with a strong deflection from -60 to $0 \mathrm{~ms}$ before response, analysis of activity correlated with net value across all participants nonetheless showed significance in this time period. Consistent with our interpretation of this response as a movementrelated potential, activity in this time window was primarily localized to sensorimotor regions, including somatosensory cortex, IPS, and premotor and motor cortices. Although this finding was unplanned, in that we did not expect to observe movement-related potentials in the
A Net Value Conjunction, Response-Locked
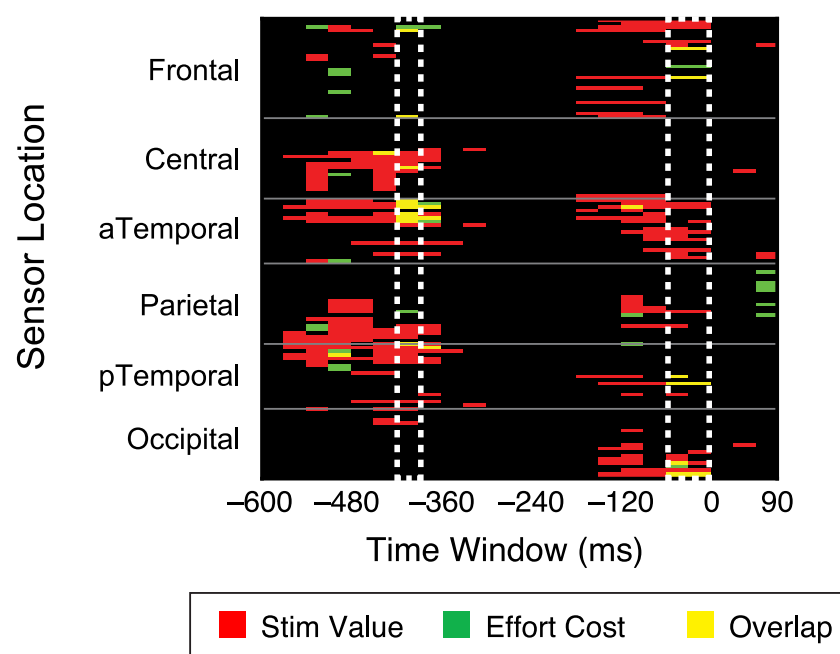

B

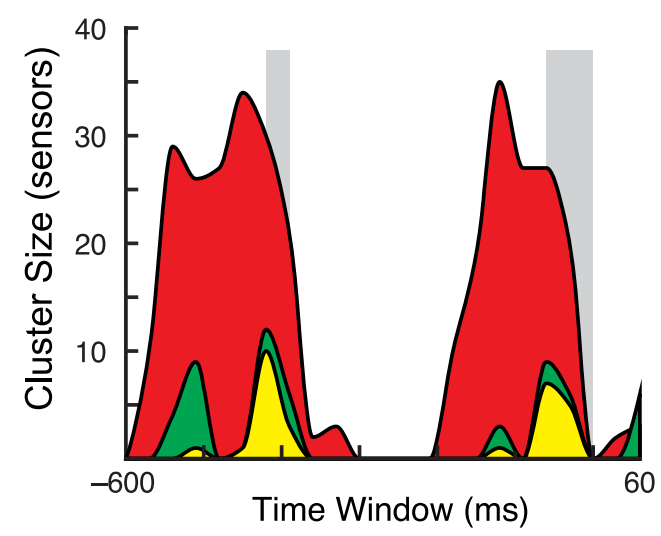

\section{Net Value Activity, -420 to -390 ms Pre-Response}

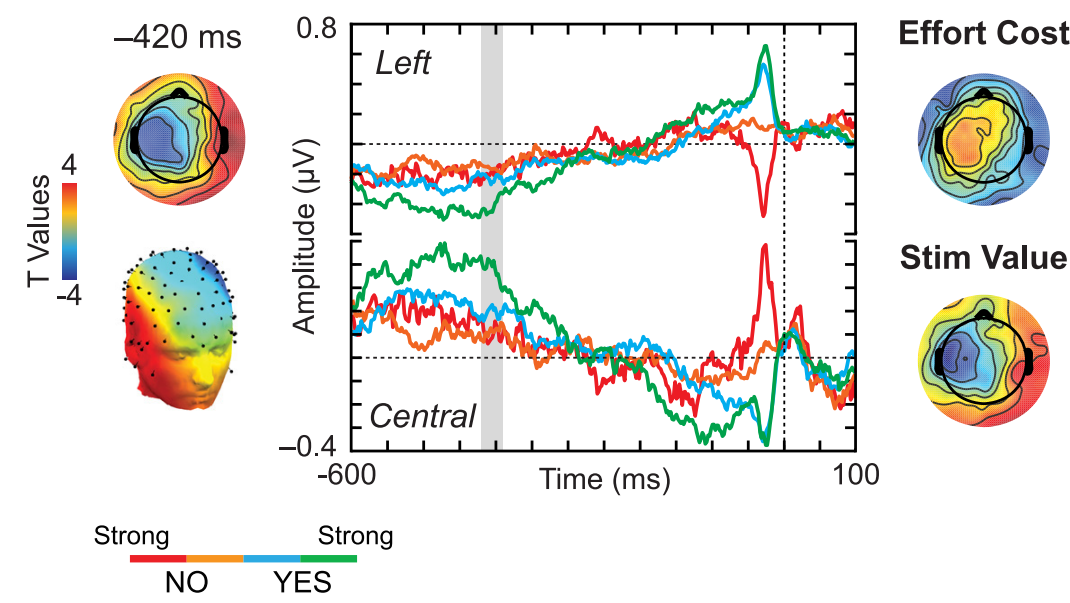

Figure 5. Net value analysis, response-locked data. $\boldsymbol{A}$, Heat map showing significant ERP activity correlated with net value, in conjunction with significant responses to stimulus value (red) and effort cost (green) alone. Clusters of overlapping activity, reflecting true net value (yellow), are seen at -420 to $-390 \mathrm{~ms}$ and -60 to $0 \mathrm{~ms}$ before response (white dashed boxes). $\boldsymbol{B}$, Cluster analysis of stimulus value, effort cost, and net value. Collapsing over scalp locations, this visualization further supports the emergence of net value signals from -420 to $-390 \mathrm{~ms}$ and -60 to $0 \mathrm{~ms}$ before response (gray boxes). C, Scalp topography and average waveforms for net value, selected using significant SOls in the -420 to $-390 \mathrm{~ms}$ time window. Scalp topography during this period shows high similarity of net value to both effort cost and stimulus value. The movement-related potential visible from -60 to $0 \mathrm{~ms}$ in this data reflects a larger proportion of motor signals recorded in one of the two groups randomly assigned to counterbalanced key mappings. 


\section{A Net Value Localization, Response-Locked}

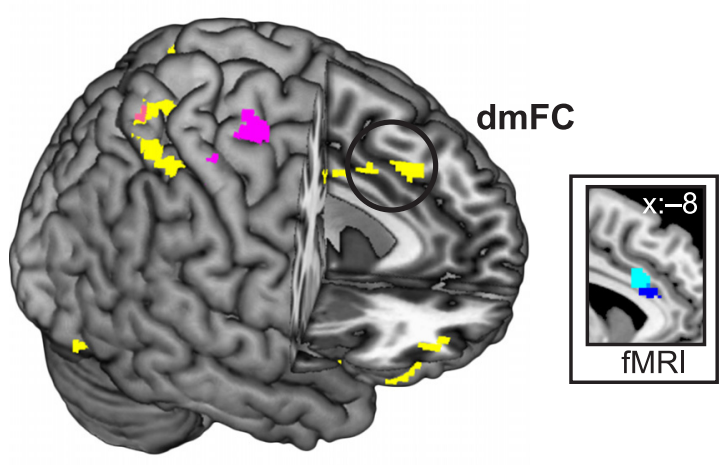

FWE $p=0.01$
-420 to $-390 \mathrm{~ms}$

-60 to $0 \mathrm{~ms}$
B Motor Response Analysis, Masked at $\mathrm{p}=0.01$
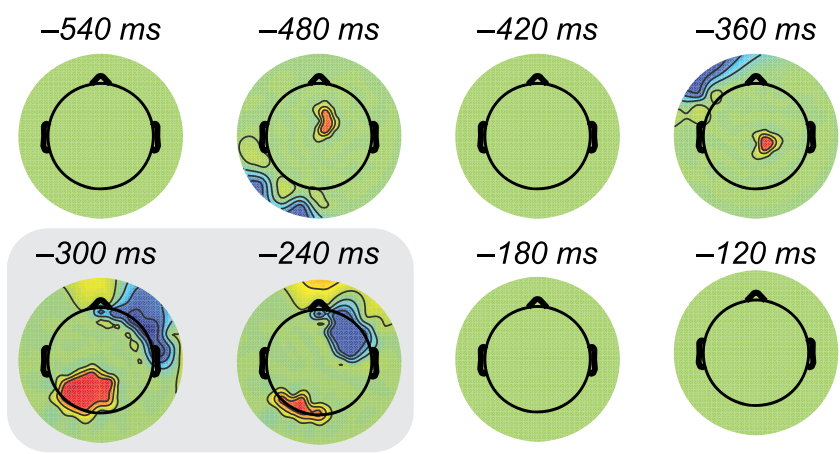

$-240 \mathrm{~ms}$
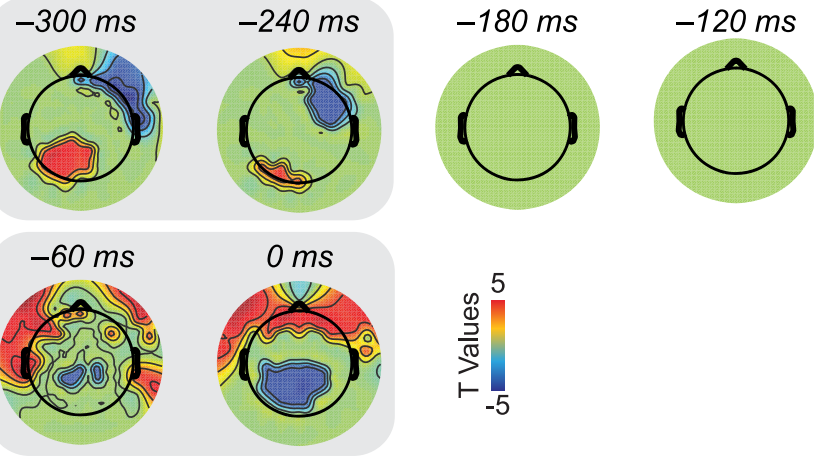

C

\section{Granger Causal Connectivity Analysis, Motor Response}

\section{-300 to -210 ms post-response}

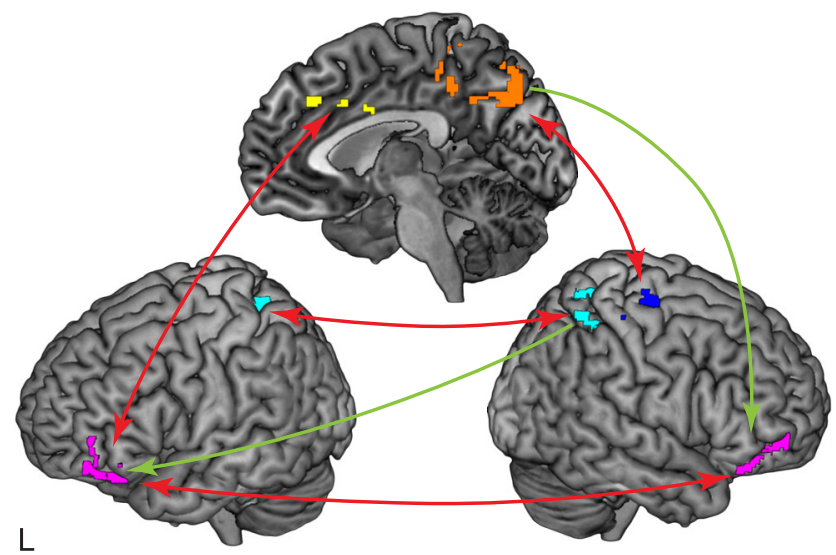

-60 to $0 \mathrm{~ms}$ post-response

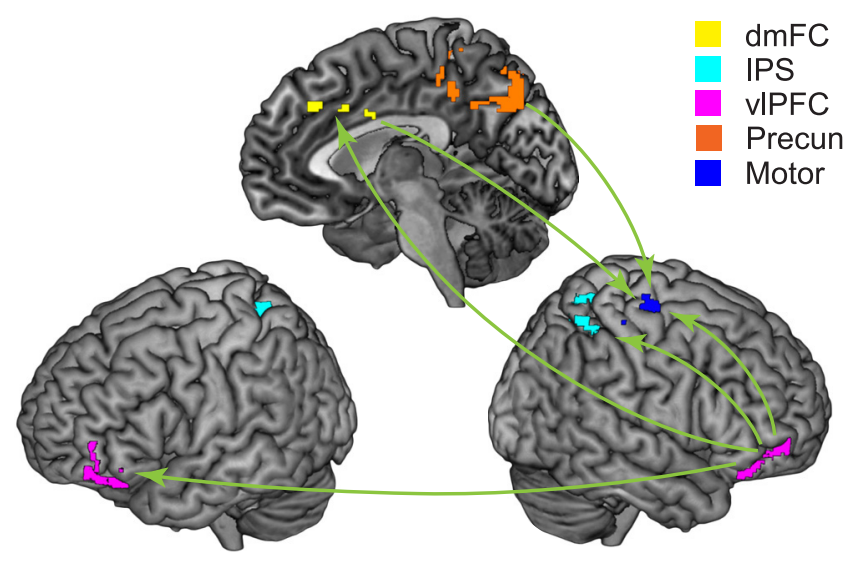

Figure 6. Net value transformation into motor output, response-locked data. $A$, Source localization of net value activity from -420 to $-390 \mathrm{~ms}$ (yellow) and -60 to $0 \mathrm{~ms}$ (magenta), FWE-corrected $p<0.01$. Inset, Spherical masks based on peak coordinates from two fMRI studies [blue, Croxson et al. (2009); magenta, Prévost et al. (2010)], 6 mm radius. $\boldsymbol{B}$, Sensors showing $t$ values associated with linear ordering by motor response (pinky, ring finger, middle finger, index finger), masked by significance at $p=0.01$. Major windows of motor activity were observed from -300 to $-210 \mathrm{~ms}$ and -60 to $0 \mathrm{~ms}$ before response (gray boxes). C, GCCA of motor response activity for the significant time windows from -300 to $-210 \mathrm{~ms}$ (left) and -60 to $0 \mathrm{~ms}$ (right) before response. Rols were selected from sensorimotor areas identified in the net value localization in $\boldsymbol{A}$; see also indicated clusters in Tables 5 and 6 . Two-headed red arrows denote bidirectional connectivity, whereas green arrows indicate unidirectional connectivity. All causal connections shown here are significant at Bonferroni-corrected $p=0.05$.

net value comparison, it provided a fortuitous opportunity to look at how sensorimotor networks associated with net value contribute to the preparation and execution of the motor response, as described below.

\section{Response-locked ERP: Granger-causal sensorimotor interactions in motor preparation}

Given the proposed role of sensorimotor networks in transforming cognitive value signals into action plans, one additional question of interest is how regions identified in the response-locked net value analysis interact to facilitate motor output. Specifically, we used the sources identified above as ROIs for an analysis of how sensorimotor networks interact in the preparation of the effector representation (pinky, ring finger, middle finger, index finger) for motor output. Because the net value comparison is defined independently of motor effector, this approach reduces the selection bias that would distort an analysis based directly on the localization of the motor response (Kriegeskorte et al., 2009).

To examine the interaction of sensorimotor networks in the time leading up to response, we used GCCA (Seth, 2010; Bressler and Seth, 2011), which allows us to map directional interactions between neural time courses reconstructed from our source localization (Harris et al., 2013). Specifically, for two simultaneously measured time courses $X$ and $Y$, time-varying signal $X$ is considered Granger causal if past information about $X$ improves prediction of $Y$ beyond what can be predicted using only lagged values of $Y$.

Because of our interest in the cortical dynamics of translating net value information into an explicit motor output, our analysis focused on the emergence of the movement-related potential in the time leading up to response. Using a linear regression with response finger (pinky, ring finger, middle finger, index finger) as 
Table 5. Peak MNI coordinates: net value, -420 to $-390 \mathrm{~ms}$ before response

\begin{tabular}{|c|c|c|c|c|c|c|}
\hline \multirow{2}{*}{$\begin{array}{l}\text { Voxels }(n) \\
178\end{array}$} & \multirow{2}{*}{$\begin{array}{l}\text { Side } \\
R\end{array}$} & \multicolumn{2}{|c|}{ Peak MNI coordinates } & & \multirow{2}{*}{$\begin{array}{l}F \\
112.8\end{array}$} & \multirow{2}{*}{$\begin{array}{l}\text { MNI coordinate region } \\
\text { IPS }^{a}\end{array}$} \\
\hline & & 20 & -54 & 58 & & \\
\hline & & 38 & -34 & 46 & 56.0 & \\
\hline & & 38 & -50 & 60 & 55.1 & \\
\hline \multirow[t]{3}{*}{86} & L & -34 & -54 & 34 & 89.4 & Angular gyrus, inferior \\
\hline & & -34 & -54 & 44 & 88.4 & parietal lobule \\
\hline & & -44 & -70 & 38 & 52.9 & \\
\hline \multirow[t]{3}{*}{145} & $\mathrm{R}$ & 32 & 56 & -2 & 88.7 & $\mathrm{vIPFC}^{a}$ \\
\hline & & 26 & 16 & -22 & 71.0 & \\
\hline & & 42 & 42 & -10 & 70.7 & \\
\hline \multirow[t]{3}{*}{244} & L & -36 & 34 & -16 & 86.1 & $\operatorname{vIPFC}^{a}$ \\
\hline & & -28 & 14 & -26 & 76.8 & \\
\hline & & -18 & 4 & -16 & 71.5 & \\
\hline \multirow[t]{3}{*}{91} & $\mathrm{R}$ & 18 & -38 & 42 & 83.3 & Precuneus \\
\hline & & 12 & -62 & 44 & 72.6 & \\
\hline & & 4 & -50 & 48 & 71.2 & \\
\hline \multirow[t]{3}{*}{359} & L & -16 & -28 & 38 & 81.4 & Precuneus $^{a}$ \\
\hline & & -6 & -68 & 36 & 79.8 & \\
\hline & & -6 & -76 & 50 & 78.3 & \\
\hline \multirow[t]{2}{*}{90} & L & -24 & -40 & 60 & 73.9 & Postcentral gyrus ${ }^{a}$ \\
\hline & & --18 & -50 & 64 & 64.0 & \\
\hline 30 & R & 18 & 44 & 24 & 71.3 & Superior frontal gyrus \\
\hline \multirow[t]{3}{*}{79} & L & -8 & 30 & 36 & 69.6 & $\mathrm{ACC}^{a}$ \\
\hline & & -10 & 14 & 34 & 54.0 & \\
\hline & & -4 & 4 & 32 & 52.0 & \\
\hline 12 & $\mathrm{R}$ & 32 & -74 & 20 & 62.4 & Middle occipital lobe \\
\hline \multirow[t]{2}{*}{24} & R & 28 & -62 & 32 & 61.4 & IPS \\
\hline & & 24 & -68 & 40 & 51.7 & \\
\hline 7 & $\mathrm{~L}$ & -8 & -6 & 30 & 51.9 & Cingulate gyrus \\
\hline
\end{tabular}

Clusters surviving FWE-corrected threshold $p<0.01(F=50.2)$ and cluster size threshold $k=5$. L, Left; $R$, right. ${ }^{a}$ Clusters used as ROls in causal connectivity analysis.

Table 6. Peak MNI coordinates: net value, -60 to $0 \mathrm{~ms}$ before response

\begin{tabular}{|c|c|c|c|c|c|c|}
\hline \multirow{2}{*}{$\begin{array}{l}\text { \# Voxels } \\
61\end{array}$} & \multirow{2}{*}{$\frac{\text { Side }}{R}$} & \multicolumn{2}{|c|}{ Peak MNI coordinates } & & \multirow{2}{*}{$\begin{array}{ll}F \\
106.9\end{array}$} & \multirow{2}{*}{$\begin{array}{l}\text { MNI coordinate region } \\
\text { Somatosensory cortex }\end{array}$} \\
\hline & & 42 & -28 & 46 & & \\
\hline & & 18 & -36 & 58 & 62.3 & \\
\hline & & 30 & -36 & 54 & 54.4 & \\
\hline \multirow[t]{2}{*}{118} & $\mathrm{R}$ & 28 & -24 & 54 & 69.4 & Motor cortex ${ }^{a}$ \\
\hline & & 26 & -16 & 70 & 68.6 & Premotor cortex ${ }^{a}$ \\
\hline \multirow[t]{3}{*}{236} & L & -12 & -48 & 28 & 65.0 & Posterior cingulate cortex \\
\hline & & -12 & -24 & 36 & 61.1 & \\
\hline & & -4 & -42 & 28 & 60.6 & \\
\hline 15 & L & -34 & -58 & 30 & 60.3 & Angular gyrus/BA 39 \\
\hline 6 & $\mathrm{~L}$ & -24 & -82 & 24 & 56.2 & Extrastriate cortex \\
\hline 10 & $\mathrm{R}$ & 22 & -56 & 62 & 52.0 & IPS \\
\hline \multirow[t]{2}{*}{20} & L & -40 & 0 & -38 & 51.7 & Anterior temporal lobe \\
\hline & & -48 & -2 & -42 & 49.8 & \\
\hline 5 & L & -38 & -70 & 42 & 50.6 & Angular gyrus/BA39 \\
\hline
\end{tabular}

Clusters surviving FWE-corrected threshold $p<0.01(F=49.3)$ and cluster size threshold $k=5$. L, Left; $\mathrm{R}$, right. ${ }^{a}$ Clusters used as ROIs in causal connectivity analysis.

the covariate, we identified sensor and time window combinations associated with a significant linear ordering from pinky to index finger. Figure $6 B$ shows scalp plots of $t$ values associated with the linear contrast on motor output, masked by significance at $p=0.01$, permutation corrected. This analysis revealed windows of significant activity associated with the finger used for motor output, emerging from -300 to $-210 \mathrm{~ms}$ and -60 to $0 \mathrm{~ms}$ before response.

For these two time windows of interest, we performed a GCCA using ROIs defined from the net value contrast. We focused on a subset of regions described above as having known associations with action planning and sensorimotor processing: left dmFC, IPS, vlPFC, precuneus, and premotor/motor cortices
(Tables 5, 6). As in previous work (Harris et al., 2013), we began by performing a Bayesian source localization of the linear contrast for motor output (pinky to index finger) for each subject. The dipole intensity maps generated by the source reconstruction were then used to forward model the projected average time course for each ROI and subject.

As shown in Figure 6C, Granger causal connectivity revealed a dynamic pattern of interaction between different motor planning areas in the time leading up to response (Bonferroni corrected, $p=0.05)$. During the time window from -300 to $-210 \mathrm{~ms}$ before response (Fig. 6C, left), strong bidirectional connections were observed throughout the selected ROIs. In addition to interhemispheric connectivity between corresponding regions of vlPFC and parietal cortex, these effects included communication between regions associated with action planning, such as precuneus and premotor/motor cortices, as well as dorsomedial PFC and vlPFC.

The latter finding is of particular interest, given the putative role of these regions in representing the value of options with respect to their potential cost (Padoa-Schioppa, 2011; Rushworth et al., 2011). Although the relatively coarse spatial resolution of EEG precludes detailed parcellation of our source reconstruction, it is notable that the ventrolateral prefrontal cluster reported here includes frontopolar coordinates previously associated with representing the value of unchosen alternatives, particularly in advance of a switch in response (Koechlin and Hyafil, 2007; Boorman et al., 2009, 2011). Consistent with an extensive literature on the role of vlPFC in changing behavioral responses to reflect the current context (Mitchell, 2011), these results suggest that vlPFC plays a role in modulating behavior to reflect environmental factors beyond the intrinsic value of the stimulus. In this view, the neuroanatomical connections of this region with premotor cortex (Carmichael and Price, 1995; Cavada et al., 2000) and with the dorsomedial regions of ACC and PFC highlighted here (Kahnt et al., 2012; Zald et al., 2014) may enable the coordination of appropriate behavioral responses given the particular biomechanical costs associated with different available actions.

During the period associated with the generation of the movement-related potential, bidirectional connections across the two hemispheres gave way to unidirectional inputs into premotor and motor cortices. In particular, the right vlPFC shows widespread connectivity during this period, making connections to left vlPFC and dmFC, right IPS, and right premotor/motor cortices. Additional inputs from dmFC and precuneus to the premotor/motor cluster are consistent with the known connectivity of these regions to the motor cortex (Paus, 2001; Margulies et al., 2009).

These results provide a window onto how stimulus values are combined with effort cost information in the time leading up to response and how the resulting net value signals are translated into motor output. Whereas the stimulus-locked analysis revealed early involvement of dorsal stream areas broadly linked to sensorimotor processing, particularly in the representation of effort cost, only in the response-locked data was net value activity localized to regions of $\mathrm{dmFC}$ and dorsal ACC implicated in action planning. Together with the connectivity among motor regions highlighted by Granger causality, these findings further shed light on how dorsomedial PFC communicates with other sensorimotor areas to transform value signals into the appropriate motor response. 


\section{Discussion}

Although tradeoffs between the intrinsic value of goods and the physical effort to obtain them are ubiquitous in daily life, researchers disagree on when and how effort cost is incorporated into decision-making at the neural level (Cisek and Kalaska, 2010; Padoa-Schioppa, 2011). Here we used ERP in the context of an effortful decision task to examine the time course and cortical dynamics of effort cost representation, as well as whether and how effort cost is integrated with stimulus value to produce a combined cost-benefit signal for optimal choice.

Behaviorally, subjects incorporated both stimulus value and physical effort cost into their decisions, as seen by the significant loadings of both liking ratings and effort level on choice. This finding was mirrored in the stimulus-locked evoked potential data, which showed neural signals correlated with effort cost and stimulus value in the time after stimulus onset. However, these data also revealed a striking disparity in the timing of these two types of processing, with effort cost activity emerging as early as $100-250 \mathrm{~ms}$ after stimulus onset, followed by stimulus value several hundred milliseconds later. Localized to traditionally sensorimotor regions, including middle cingulate, somatosensory, and motor cortices, this early effort cost activity is consistent with ethologically inspired models that posit rapid involvement of sensorimotor networks in the decision process, such as the affordance competition hypothesis (Cisek and Kalaska, 2010). Thus, effort cost can be encoded from early latencies, at least when this information is relevant to choice. Standard methodological approaches based on simple key-press responses or highly trained, stereotyped movements may underestimate the role of sensorimotor networks during real-world decision-making, highlighting the importance of ecological validity in experimental design.

These results also extend parallel models of choice by demonstrating a cognitive representation of effort cost in areas including vmPFC, in line with the good-based model (Padoa-Schioppa, 2011). Emerging 450-600 ms after stimulus onset, this vmPFC source overlapped with those identified for both stimulus value and the combined net value parameter. Although our experimental design could not distinguish which specific aspects of effort cost information (e.g., visual stimulus, haptic information, action preparation) were represented in the vmPFC value signal, these results suggest that information about physical exertion may be incorporated into cognitive representations of value. This idea is consistent with recent results demonstrating that anticipated sensorimotor contingencies can reduce the likelihood of changes of mind in perceptual decisionmaking, dynamically influencing choice selection (Burk et al., 2014; Moher and Song, 2014).

Along with previous ERP data (Harris et al., 2011, 2013), our results suggest a timeframe for the integration of subjective value signals from multiple types of information within vmPFC. However, this window was substantially later than that seen in singleunit recordings, which often find effects of value by $200 \mathrm{~ms}$ (Kennerley et al., 2011). Although human ERP components tend to be slower than their macaque homologs (Woodman, 2012), another relevant aspect of animal studies is the substantially higher degree of behavioral training before recording (e.g., 600-1200 trials; Kennerley et al., 2009), often coupled with the use of relatively few and/or simplified stimuli. Similarly, we have observed additional value signals emerging as early as 150-250 $\mathrm{ms}$ after stimulus over repeated stimulus presentations (Harris et al., 2011) and multiple ERP recording sessions (Harris et al.,
2013). Differences in stimulus complexity may likewise explain why we found slower integration of net value than a recent study of biomechanical effort cost (Cos et al., 2014). In this light, it may be warranted to control for the visual complexity of the stimulus options relative to the effort cue in future experiments, for example by representing stimuli using simplified tokens (e.g., colored squares).

Therefore, these data suggest that the representation of effort cost reflects elements of both the serial information processing model and affordance competition hypothesis, with rapid and persistent engagement of sensorimotor networks followed by activity in cognitive valuation regions such as vmPFC. Although previous work has suggested how good-based and action-based representations might be combined (Cisek, 2012; Cai and PadoaSchioppa, 2014), our results provide new evidence regarding the cortical dynamics of this process.

Examining the response-locked data, we found continued evidence for the dynamic interaction of sensorimotor and cognitive systems in the time leading up to motor output. Net value responses were identified in the response-locked data from -420 to $-390 \mathrm{~ms}$ before response, $\sim 100 \mathrm{~ms}$ before the first time window significantly associated with motor response output. Based on our distributed source localization, this process appears to involve sensorimotor regions beyond the aforementioned ACC and $\mathrm{dmFC}$, including areas of posterior parietal cortex, precuneus, and vlPFC. Whereas an extensive literature in both humans and animals has linked the posterior parietal lobe to visually guided action (for review, see Creem and Proffitt, 2001), the functions of the human precuneus are less well defined and likely reflect discrete functional subdivisions (Margulies et al., 2009). Compounding this difficulty is the coarse spatial precision of EEG, which precludes fine-grained demarcation of our sources. Nonetheless, it is worth noting that resting state functional connectivity analyses have found connections between specific subregions of precuneus and components of the dorsal sensorimotor stream, including posterior parietal cortex and premotor cortex (Margulies et al., 2009).

In this context, the localization of activity to vlPFC is of particular interest, because this area is not traditionally considered part of the dorsal sensorimotor stream. Nevertheless, this region has been implicated in changing behavior depending on the environmental context, including the inhibition of motor responses in stop-signal and go/no-go tasks (for review, see Mitchell, 2011). Consistent with this idea, neuroanatomical tracing (Carmichael and Price, 1995; Cavada et al., 2000) and functional connectivity (Kahnt et al., 2012; Zald et al., 2014) studies have found connections between lateral PFC and components of the motor circuit, including dmFC and middle cingulate cortex, as well as parts of premotor cortex. At the same time, activations in vlPFC and nearby frontopolar PFC have been found for purely cognitive processes, including attentional control (Hampshire et al., 2010), integration of relational information during reasoning (Christoff et al., 2001), and representation of counterfactual (unchosen) outcomes (Boorman et al., 2009, 2011).

Collectively, these data support a role for vlPFC in coordination of multiple mental operations across different rules or domains, including cognition and action (Ramnani and Owen, 2004; Koechlin and Hyafil, 2007). In line with this idea, we found that this region was linked not only to the representation of net value in the response-locked data but also stimulus value and effort cost in the stimulus-locked analysis. Likewise, GCCA showed persistent and extensive connectivity between bilateral regions of vlPFC and between vlPFC and action planning regions 
in the time leading up to motor output. Although Granger connectivity is not sufficient to establish true causality, these results complement the aforementioned literature by providing highresolution temporal information regarding how and when vlPFC contributes to the transformation of abstract cognitive information into action plans.

A number of questions remain regarding the coding of cost and benefit in the brain. For example, recent research suggests that neural populations within specific subregions of ACC encode cost and benefit in an opponent manner, with some neurons responding selectively to the magnitude of an aversive event (air puff) that was paired with an appetitive reward (Amemori and Graybiel, 2012). Critically, microstimulation of this area resulted in persistent increases in avoidance behavior, whereas administration of anti-anxiety medication reversed this effect. These results highlight the intersection of decision-making with emotion and arousal, in line with the known roles of ACC and dmFC in emotional regulation (Mitchell, 2011). The cumulative nature of the microstimulation effect also points to how net value computations can be affected by individual differences in the perceived aversiveness of cost, both at the trait level and as a result of pathological states, such as depression and anxiety. Although here we manipulated the positive motivational significance of the food stimulus by requiring subjects to fast before the experiment, additional studies could examine how factors such as anxiety and physiological stress modulate the negative representation of cost. Given our findings regarding the temporal dynamics of physical effort cost representation, one question of particular interest is whether the estimation of cost measured in ACC by Amemori and Graybiel (2012) emerges solely during net value computation or reflects changing biases in rapid sensorimotor encoding from early in the trial.

In summary, these data extend current understanding of when and how sensorimotor networks compute physical effort cost and integrate this information into a net value signal for decision-making. Bridging two theoretical models of decisionmaking, serial information processing versus the affordance competition hypothesis, our results highlight the flexible and dynamic engagement of sensorimotor areas across the time course of choice. Additional research examining how cost estimation is influenced by task demands, individual differences, and pathological states may have broad implications for understanding how the interaction of intrinsic preferences and motor affordances leads to complex and varied cost-benefit valuations in real-world scenarios.

\section{References}

Amemori K, Graybiel AM (2012) Localized microstimulation of primate pregenual cingulate cortex induces negative decision-making. Nat Neurosci 15:776-785. CrossRef Medline

Belouchrani A, Abed-Meraim K, Cardoso JF, Moulines E (1997) A blind source separation technique using second-order statistics. IEEE Trans Signal Process 45:434-444. CrossRef

Boorman ED, Behrens TE, Woolrich MW, Rushworth MF (2009) How green is the grass on the other side? Frontopolar cortex and the evidence in favor of alternative courses of action. Neuron 62:733-743. CrossRef Medline

Boorman ED, Behrens TE, Rushworth MF (2011) Counterfactual choice and learning in a neural network centered on human lateral frontopolar cortex. PLoS Biol 9:e1001093. CrossRef Medline

Brainard DH (1997) The Psychophysics Toolbox. Spatial Vis 10:433-436. CrossRef

Bressler SL, Seth AK (2011) Wiener-Granger causality: a well established methodology. Neuroimage 58:323-329. CrossRef Medline

Burk D, Ingram JN, Franklin DW, Shadlen MN, Wolpert DM (2014) Motor effort alters changes of mind in sensorimotor decision making. PLoS One 9:e92681. CrossRef Medline

Burke CJ, Brünger C, Kahnt T, Park SQ, Tobler PN (2013) Neural integration of risk and effort costs by the frontal pole: only upon request. J Neurosci 33:1706-1713a. CrossRef Medline

Cai X, Padoa-Schioppa C (2014) Contributions of orbitofrontal and lateral prefrontal cortices to economic choice and the good-to-action transformation. Neuron 81:1140-1151. CrossRef Medline

Carmichael ST, Price JL (1995) Sensory and premotor connections of the orbital and medial prefrontal cortex of macaque monkeys. J Comp Neurol 363:642-664. CrossRef Medline

Cavada C, Compañy T, Tejedor J, Cruz-Rizzolo RJ, Reinoso-Suárez F (2000) The anatomical connections of the macaque monkey orbitofrontal cortex. A review. Cereb Cortex 10:220-242. CrossRef Medline

Christoff K, Prabhakaran V, Dorfman J, Zhao Z, Kroger JK, Holyoak KJ, Gabrieli JD (2001) Rostrolateral prefrontal cortex involvement in relational integration during reasoning. Neuroimage 14:1136-1149. CrossRef Medline

Cisek P (2012) Making decisions through a distributed consensus. Curr Opin Neurobiol 22:927-936. CrossRef Medline

Cisek P, Kalaska JF (2005) Neural correlates of reaching decisions in dorsal premotor cortex: specification of multiple direction choices and final selection of action. Neuron 45:801-814. CrossRef Medline

Cisek P, Kalaska JF (2010) Neural mechanisms for interacting with a world full of action choices. Annu Rev Neurosci 33:269-298. CrossRef Medline

Clithero JA, Rangel A (2014) Informatic parcellation of the network involved in the computation of subjective value. Soc Cogn Affect Neurosci 9:1289-1302. CrossRef Medline

Cohen MX, Cavanagh JF, Slagter HA (2011) Event-related potential activity in the basal ganglia differentiates rewards from nonrewards: temporospatial principal components analysis and source localization of the feedback negativity: commentary. Hum Brain Mapp 32:2270-2271. CrossRef Medline

Cos I, Duque J, Cisek P (2014) Rapid prediction of biomechanical costs during action decisions. J Neurophysiol 112:1256-1266. CrossRef Medline

Craig AD (2002) How do you feel? Interoception: the sense of the physiological condition of the body. Nat Rev Neurosci 3:655-666. CrossRef Medline

Creem SH, Proffitt DR (2001) Defining the cortical visual systems: "what," "where," and "how." Acta Psychol (Amst) 107:43-68. CrossRef Medline

Croxson PL, Walton ME, O’Reilly JX, Behrens TE, Rushworth MF (2009) Effort-based cost-benefit valuation and the human brain. J Neurosci 29: 4531-4541. CrossRef Medline

Delorme A, Makeig S (2004) EEGLAB: an open source toolbox for analysis of single-trial EEG dynamics including independent component analysis. J Neurosci Methods 134:9-21. CrossRef Medline

Dijkerman HC, de Haan EH (2007) Somatosensory processes subserving perception and action. Behav Brain Sci 30:189-201; discussion 201-239. CrossRef Medline

Di Russo F, Martínez A, Sereno MI, Pitzalis S, Hillyard SA (2002) Cortical sources of the early components of the visual evoked potential. Hum Brain Mapp 15:95-111. CrossRef Medline

Fort A, Besle J, Giard MH, Pernier J (2005) Task-dependent activation latency in human visual extrastriate cortex. Neurosci Lett 379:144-148. CrossRef Medline

Friston K, Harrison L, Daunizeau J, Kiebel S, Phillips C, Trujillo-Barreto N, Henson R, Flandin G, Mattout J (2008) Multiple sparse priors for the M/EEG inverse problem. Neuroimage 39:1104-1120. CrossRef Medline

Hampshire A, Chamberlain SR, Monti MM, Duncan J, Owen AM (2010) The role of the right inferior frontal gyrus: inhibition and attentional control. Neuroimage 50:1313-1319. CrossRef Medline

Hare TA, O'Doherty J, Camerer CF, Schultz W, Rangel A (2008) Dissociating the role of the orbitofrontal cortex and the striatum in the computation of goal values and prediction errors. J Neurosci 28:5623-5630. CrossRef Medline

Hare TA, Camerer CF, Rangel A (2009) Self-control in decision-making involves modulation of the vmPFC valuation system. Science 324:646-648. CrossRef Medline

Harris A, Adolphs R, Camerer C, Rangel A (2011) Dynamic construction of stimulus values in the ventromedial prefrontal cortex. PLoS One 6:e21074. CrossRef Medline

Harris A, Hare T, Rangel A (2013) Temporally dissociable mechanisms of 
self-control: early attentional filtering versus late value modulation. J Neurosci 33:18917-18931. CrossRef Medline

Henson RN, Mattout J, Singh KD, Barnes GR, Hillebrand A, Friston K (2007) Population-level inferences for distributed MEG source localization under multiple constraints: application to face-evoked fields. Neuroimage 38:422-438. CrossRef Medline

Holmes MD (2008) Dense array EEG: Methodology and new hypothesis on epilepsy syndromes. Epilepsia 49:3-14. CrossRef Medline

Jeannerod M (2001) Neural simulation of action: a unifying mechanism for motor cognition. Neuroimage 14:S103-S109. CrossRef Medline

Jung TP, Makeig S, Humphries C, Lee TW, McKeown MJ, Iragui V, Sejnowski TJ (2000) Removing electroencephalographic artifacts by blind source separation. Psychophysiology 37:163-178. CrossRef Medline

Kable JW, Glimcher PW (2009) The neurobiology of decision: consensus and controversy. Neuron 63:733-745. CrossRef Medline

Kahnt T, Chang LJ, Park SQ, Heinzle J, Haynes JD (2012) Connectivitybased parcellation of the human orbitofrontal cortex. J Neurosci 32: 6240-6250. CrossRef Medline

Kennerley SW, Dahmubed AF, Lara AH, Wallis JD (2009) Neurons in the frontal lobe encode the value of multiple decision variables. J Cogn Neurosci 21:1162-1178. CrossRef Medline

Kennerley SW, Behrens TE, Wallis JD (2011) Double dissociation of value computations in orbitofrontal and anterior cingulate neurons. Nat Neurosci 14:1581-1589. CrossRef Medline

Koechlin E, Hyafil A (2007) Anterior prefrontal function and the limits of human decision-making. Science 318:594-598. CrossRef Medline

Kranczioch C, Mathews S, Dean PJ, Sterr A (2009) On the equivalence of executed and imagined movements: evidence from lateralized motor and nonmotor potentials. Hum Brain Mapp 30:3275-3286. CrossRef Medline

Kriegeskorte N, Simmons WK, Bellgowan PS, Baker CI (2009) Circular analysis in systems neuroscience: the dangers of double dipping. Nat Neurosci 12:535-540. CrossRef Medline

Litt A, Plassmann H, Shiv B, Rangel A (2011) Dissociating valuation and saliency signals during decision-making. Cereb Cortex 21:95-102. CrossRef Medline

Litvak V, Friston K (2008) Electromagnetic source reconstruction for group studies. Neuroimage 42:1490-1498. CrossRef Medline

Luck SJ (2005) An introduction to the event-related potential technique. Cambridge, MA: Massachusetts Institute of Technology.

Margulies DS, Vincent JL, Kelly C, Lohmann G, Uddin LQ, Biswal BB, Villringer A, Castellanos FX, Milham MP, Petrides M (2009) Precuneus shares intrinsic functional architecture in humans and monkeys. Proc Natl Acad Sci U S A 106:20069-20074. CrossRef Medline

Mitchell DG (2011) The nexus between decision making and emotion regulation: a review of convergent neurocognitive substrates. Behav Brain Res 217:215-231. CrossRef Medline

Moher J, Song JH (2014) Perceptual decision processes flexibly adapt to avoid change-of-mind motor costs. J Vis 14:1. CrossRef Medline

Padoa-Schioppa C (2011) Neurobiology of economic choice: a good-based model. Annu Rev Neurosci 34:333-359. CrossRef Medline

Paus T (2001) Primate anterior cingulate cortex: where motor control, drive and cognition interface. Nat Rev Neurosci 2:417-424. CrossRef Medline

Plassmann H, O’Doherty J, Rangel A (2007) Orbitofrontal cortex encodes willingness to pay in everyday economic transactions. J Neurosci 27: 9984-9988. CrossRef Medline
Prévost C, Pessiglione M, Météreau E, Cléry-Melin ML, Dreher JC (2010) Separate valuation subsystems for delay and effort decision costs. J Neurosci 30:14080-14090. CrossRef Medline

Ramnani N, Owen AM (2004) Anterior prefrontal cortex: insights into function from anatomy and neuroimaging. Nat Rev Neurosci 5:184-194. CrossRef Medline

Rangel A, Hare T (2010) Neural computations associated with goal-directed choice. Curr Opin Neurobiol 20:262-270. CrossRef Medline

Rushworth MF, Behrens TE, Rudebeck PH, Walton ME (2007) Contrasting roles for cingulate and orbitofrontal cortex in decisions and social behaviour. Trends Cogn Sci 11:168-176. CrossRef Medline

Rushworth MF, Mars RB, Summerfield C (2009) General mechanisms for making decisions? Curr Opin Neurobiol 19:75-83. CrossRef Medline

Rushworth MF, Noonan MP, Boorman ED, Walton ME, Behrens TE (2011) Frontal cortex and reward-guided learning and decision-making. Neuron 70:1054-1069. CrossRef Medline

Seth AK (2010) A MATLAB toolbox for Granger causal connectivity analysis. J Neurosci Methods 186:262-273. CrossRef Medline

Song JH, Nakayama K (2009) Hidden cognitive states revealed in choice reaching tasks. Trends Cogn Sci 13:360-366. CrossRef Medline

Sternberg S (1969) The discovery of processing stages: extensions of Donders' method. Acta Psychol (Amst) 30:276-315. CrossRef

Tang AC, Sutherland MT, McKinney CJ (2005) Validation of SOBI components from high-density EEG. Neuroimage 25:539-553. CrossRef Medline

Taylor MJ (2002) Non-spatial attentional effects on P1. Clin Neurophysiol 113:1903-1908. CrossRef Medline

Thomas NW, Paré M (2007) Temporal processing of saccade targets in parietal cortex area LIP during visual search. J Neurophysiol 97:942-947. CrossRef Medline

Toro C, Deuschl G, Thatcher R, Sato S, Kufta C, Hallett M (1994) Eventrelated desynchronization and movement-related cortical potentials on the ECoG and EEG. Electroencephalogr Clin Neurophysiol 93:380-389. CrossRef Medline

Urbano A, Babiloni C, Onorati P, Carducci F, Ambrosini A, Fattorini L, Babiloni F (1998) Responses of human primary sensorimotor and supplementary motor areas to internally triggered unilateral and simultaneous bilateral one-digit movements. A high-resolution EEG study. Eur J Neurosci 10:765-770. CrossRef Medline

Wager TD, Keller MC, Lacey SC, Jonides J (2005) Increased sensitivity in neuroimaging analyses using robust regression. Neuroimage 26:99-113. CrossRef Medline

Woodman GF (2012) Homologues of human ERP components in nonhuman primates. In: The Oxford handbook of event-related potential components (Luck SJ, Kappenman ES, eds), pp 611-626. New York: Oxford UP.

Wunderlich K, Rangel A, O’Doherty JP (2009) Neural computations underlying action-based decision making in the human brain. Proc Natl Acad Sci U S A 106:17199-17204. CrossRef Medline

Yarkoni T, Poldrack RA, Nichols TE, Van Essen DC, Wager TD (2011) Large-scale automated synthesis of human functional neuroimaging data. Nat Methods 8:665-670. CrossRef Medline

Zald DH, McHugo M, Ray KL, Glahn DC, Eickhoff SB, Laird AR (2014) Meta-analytic connectivity modeling reveals differential functional connectivity of the medial and lateral orbitofrontal cortex. Cereb Cortex 24:232-248. CrossRef Medline 